\title{
Theory of the intermediate tilted smectic phases and their helical rotation
}

\author{
A. V. Emelyanenko, ${ }^{1,3, *}$ Atsuo Fukuda, ${ }^{2,3}$ and J. K. Vij ${ }^{3}$ \\ ${ }^{1}$ Department of Physics, Moscow State University, Moscow 119992, Russia \\ ${ }^{2}$ Department of Environmental Materials Science, Tokyo Denki University, Tokyo 101-8457, Japan \\ ${ }^{3}$ Department of Electronic and Electrical Engineering, Trinity College, University of Dublin, Dublin 2, Ireland
}

(Received 7 April 2005; revised manuscript received 10 January 2006; published 13 July 2006)

\begin{abstract}
A molecular-statistical theory for the entire sequence of the chiral tilted smectic phases is derived. Uniaxial and biaxial subphases were found to be stable in different temperature ranges depending on the molecular parameters. The model of a chiral molecule possessing a strong transverse terminal dipole moment and a quadrupole moment located in the molecular core was used. Direct dispersion and electrostatic interactions (modulated by shape) between molecules located in the same or in the neighboring smectic layers are taken into account. An effective long-range interaction arises after the minimization of the free energy with respect to polarization vectors. If the molecular quadrupole moment is small, only uniaxial phases with different periodicities arise. Their periodicity may be tens and hundreds of layers $\left(\mathrm{Sm}-C^{*}\right)$, or approximately two layers $\left(\mathrm{Sm}-C_{A}^{*}\right)$, or several layers $\left(\mathrm{Sm}-C_{\alpha}^{*}\right)$. In the presence of the nonpolar biaxial ordering (in addition to polarization) there is a cap-shaped border in the phase diagram that separates Sm- $C_{A}^{*}, \mathrm{Sm}-C^{*}$, and $\mathrm{Sm}-C_{\alpha^{*}}^{*}$ If the molecules are nonchiral, Sm- $C_{A}, \mathrm{Sm}-C$, and the de Vries's phases arise instead of the three phases mentioned above. If the molecular quadrupole moment is large, the left "arm" of the border breaks into two lines, and a sequence of biaxial subphases arises in the area between these two lines. Among these biaxial subphases, the one with periodicity of three smectic layers appears to be the broadest in the temperature range. In addition, the subphases with different periodicities were found to be stable in narrow temperature ranges. The long helical rotation in every biaxial subphase is calculated. It is found to change sign between the three-layer subphase and Sm- $C^{*}$, and may diverge in the four-layer subphase if it arises. All calculations are done with help of (A)FLC Phase Diagram Plotter software developed by the first author and available at his web-page.
\end{abstract}

DOI: 10.1103/PhysRevE.74.011705

PACS number(s): $61.30 . \mathrm{Cz}, 64.70 . \mathrm{Md}$

\section{INTRODUCTION}

In our previous theoretical papers [1,2] we have shown that a simple model based on the discrete flexoelectric polarization [3-5] can describe the entire sequence of biaxial subphases. Hereafter we shall use the definition $\mathrm{Sm}-C_{A}^{*}\left(q_{T}\right)$ for the biaxial subphases introduced in our experimental papers $[6,7]$. Parameter $q_{T}$ denoting the number of synclinic ferroelectric orderings per one short period of particular phase was introduced for the first time in [8,9]. It varies from zero in $\mathrm{Sm}-C_{A}^{*}$ to one in $\mathrm{Sm}-C^{*}$. According to this classification the three- and four-layer biaxial subphases (which are sometimes called FI1 and FI2) may be defined as Sm- $C_{A}^{*}(1 / 3)$ and $\mathrm{Sm}-C_{A}^{*}(1 / 2)$, respectively. Since the biaxial subphase structure was assumed to be commensurate, i.e., to repeat itself in a period of several smectic layers, however, the previous papers $[1,2]$ could not deal with the macroscopic helical structure of $\operatorname{Sm}-C_{A}^{*}\left(q_{T}\right)$. In this paper we are going to advance the previous theoretical treatment and to calculate the macroscopic helical pitch in every phase using a perturbation scheme.

Here we are also going to generalize our theory for the case of uniaxial $\mathrm{Sm}-C_{\alpha}^{*}$ subphase observed between $\mathrm{Sm}-C^{*}$ and $\mathrm{Sm}-A^{*}$, and thus, the entire sequence of the chiral tilted smectic phases (including the fundamental ones, $\mathrm{Sm}-C_{A}^{*}$ and $\mathrm{Sm}-C^{*}$, as well as the uniaxial and biaxial subphases) will be described. Although several preliminary phase diagrams

*URL: http://polly.phys.msu.ru/ $/$ emel/ were already presented in our experimental papers [6,7], theoretical explanation for them will be given in this paper for the first time. Several discrepancies in the previous diagrams will be resolved by taking into account the new effects.

One of the discrepancies concerns an abrupt transition between $\mathrm{Sm}-C^{*}$ and $\mathrm{Sm}-C_{\alpha}^{*}$. Our preliminary diagrams [6] always demonstrated a continuous modification from $\mathrm{Sm}-C^{*}$ to $\mathrm{Sm}-C_{\alpha}^{*}$, whereas experimentally both an abrupt transition and a continuous modification are possible. An effect related to a presence of the nonpolar biaxial ordering of molecules will be considered to describe the first-order phase transition between $\mathrm{Sm}-C^{*}$ and $\mathrm{Sm}-C_{\alpha}^{*}$. It will also be shown that in the case of nonchiral molecules the same effect may lead to the appearance of the de Vries's phase [10].

Another discrepancy which is going to be resolved in this paper is that experimentally the temperature range of $\mathrm{Sm}-C_{A}^{*}(1 / 2)$ is usually smaller than that of $\mathrm{Sm}-C_{A}^{*}(1 / 3)$, whereas in our previous theory $[1,2]$ the situation was just the opposite, and generally the temperature range of the biaxial subphase was found to decrease with $q_{T}$ decreasing. Here an effect related to the presence of the vertical (perpendicular to the smectic layer plane) flexoelectric polarization will be found responsible for the suppression of $\mathrm{Sm}-C_{A}^{*}(1 / 2)$. This polarization is proportional to the additional power of the tilt angle with respect to the considered previously (parallel to the smectic layer plane) flexoelectric polarization. It exists only in the biaxial subphases and is equal to zero in the uniaxial phases $\left(\mathrm{Sm}-C_{A}^{*}, \mathrm{Sm}-C^{*}\right.$, and $\left.\mathrm{Sm}-C_{\alpha}^{*}\right)$. The four-layer biaxial subphase $\left[\mathrm{Sm}-C_{A}^{*}(1 / 2)\right]$ appears to be suppressed completely if the tilt angle and, con- 
sequently, the vertical flexoelectric polarization is large (MHPOBC, MHDDOPTCOB) $[11,12]$. At the same time, the temperature range of $\mathrm{Sm}-C_{A}^{*}(1 / 2)$ in $10 \mathrm{OTBBB} 1 \mathrm{M} 7$ and MHPBC is very broad, where the tilt angle is small.

Recently the materials were synthesized where neither $\mathrm{Sm}-C_{A}^{*}(1 / 2)$ nor $\mathrm{Sm}-C_{A}^{*}(1 / 3)$ is observed, but the biaxial subphase different from $\mathrm{Sm}-C_{A}^{*}(1 / 2)$ and $\mathrm{Sm}-C_{A}^{*}(1 / 3)$ arises in between $\mathrm{Sm}-C_{A}^{*}(0)$ and $\mathrm{Sm}-C_{\alpha}^{*}[7]$. The most important molecular parameters that influence the phase diagram in our theory are the terminal transverse dipole moment (associated with an appropriate polar bond in the molecular tail) and the central quadrupole moment (associated with the aromatic rings in the molecular core). It will be shown in this paper that the phase sequences without the presence of $\mathrm{Sm}-C_{A}^{*}(1 / 2)$ and $\mathrm{Sm}-C_{A}^{*}(1 / 3)$ and still exhibiting different biaxial subphases are possible in the case of the large molecular dipole and quadrupole moments.

The main purpose of this paper is to obtain the phase diagrams which can reproduce with minimal discrepancy the phase sequences experimentally observed, to identify the molecular parameters responsible for the various phase sequences, to clarify the macroscopic helical structure of the biaxial subphases $\mathrm{Sm}-C_{A}^{*}\left(q_{T}\right)$, and to explain an abrupt transition between $\mathrm{Sm}-C^{*}$ and $\mathrm{Sm}-C_{\alpha}^{*}$. In Sec. II we will begin with consideration of the free energy of the perfect smectic state composed of biaxial molecules that have only freedom of rotation around their long axes. In Sec. III we will derive a perturbation theory describing the macroscopic helical rotation in an arbitrary chiral tilted smectic phase. In Sec. IV the biaxial nonpolar ordering of molecules will be considered and an abrupt transition from Sm- $C^{*}$ to $\mathrm{Sm}-C_{\alpha}^{*}$ (or from $\mathrm{Sm}-C$ to the de Vries's phase) will be explained. In Sec. V the general phase diagrams will be presented and discussed. Finally, in Sec. VI our conclusions will be made.

\section{FREE ENERGY OF THE TILTED SMECTIC STATE}

\section{A. Some basic points of the statistical theory}

The free energy of the perfect smectic state composed of biaxial molecules, that have only freedom of rotation around their long axes, can be written in the following form:

$$
\begin{aligned}
F= & \rho k_{B} T \sum_{i=1}^{N} \int f_{i}\left(\psi_{1}\right) \ln f_{i}\left(\psi_{1}\right) d \psi_{1} \\
& -\frac{1}{2} \rho^{2} k_{B} T \sum_{i, j=1}^{N} \int f_{i}\left(\psi_{1}\right) f_{j}\left(\psi_{2}\right) g_{i j}\left(\mathbf{r}_{\perp}\right) \\
& \times\left[\exp \left(-\frac{U_{i j}}{k_{B} T}\right)-1\right] d^{2} \mathbf{r}_{\perp} d \psi_{1} d \psi_{2},
\end{aligned}
$$

where $N$ is the total number of smectic layers, $\rho$ is the density of molecules inside layers, $f_{i}(\psi)$ is the distribution function for the orientation of the transverse dipole moment $\boldsymbol{\mu}$ of a molecule located in layer $i$ specified by the angle $\psi, U_{i j}$ is the total interaction potential for a molecule in layer $i$ and a molecule in layer $j$, function $g_{i j}\left(\mathbf{r}_{\perp}\right)$ describes positional correlations between molecules 1 and 2 located in layers $i$ and $j$, respectively, and vector $\mathbf{r}_{\perp}$ is a projection of the intermolecular vector $\mathbf{r}$ on the smectic layer plane. The first term in Eq. (1) is the orientational entropy and the second term is the internal energy. Minimization of the free energy (1) yields the following expression for the orientational distribution function:

$$
f_{i}\left(\psi_{1}\right)=\frac{1}{Z_{i}} \exp \left\{-\rho \beta U_{M F}^{(i)}\left(\psi_{1}\right)\right\},
$$

where $\beta \equiv 1 /\left(k_{B} T\right)$,

$$
Z_{i}=\int_{0}^{2 \pi} \exp \left\{-\rho \beta U_{M F}^{(i)}\left(\psi_{1}\right)\right\} d \psi_{1},
$$

and where $U_{M F}^{(i)}(\psi)$ is the "mean field" for a molecule in layer $i$ :

$$
\begin{aligned}
U_{M F}^{(i)}\left(\psi_{1}\right) \equiv & -k_{B} T \sum_{j=1}^{N} \int f_{j}\left(\psi_{2}\right) g_{i j}\left(\mathbf{r}_{\perp}\right) \\
& \times\left[\exp \left(-\frac{U_{i j}}{k_{B} T}\right)-1\right] d^{2} \mathbf{r}_{\perp} d \psi_{2} .
\end{aligned}
$$

Substituting the distribution function (2) back into Eq. (1), one obtains the following expression for the free energy of layer $i$ depending on the orientation of the short molecular axes:

$$
F_{i}=-\rho k_{B} T \ln Z_{i}-\frac{1}{2} \rho^{2} \int_{0}^{2 \pi} f_{i}\left(\psi_{1}\right) U_{M F}^{(i)}\left(\psi_{1}\right) d \psi_{1} .
$$

Now let us expand $\ln Z_{i}$ in Taylor series with respect to $U_{M F}^{(i)}\left(\psi_{1}\right)$ up to the square term using Eq. (3) and neglecting the constant term:

$$
\begin{aligned}
F_{i}= & \frac{\rho^{2}}{2 \pi} \int_{0}^{2 \pi}\left\{U_{M F}^{(i)}\left(\psi_{1}\right)-\frac{\rho}{2 k_{B} T}\left[U_{M F}^{(i)}\left(\psi_{1}\right)\right]^{2}\right\} d \psi_{1} \\
& +\frac{\rho^{3}}{8 \pi^{2} k_{B} T}\left[\int_{0}^{2 \pi} U_{M F}^{(i)}\left(\psi_{1}\right) d \psi_{1}\right]^{2}-\frac{1}{2} \rho^{2} \int_{0}^{2 \pi} f_{i}\left(\psi_{1}\right) U_{M F}^{(i)} \\
& \times\left(\psi_{1}\right) d \psi_{1} .
\end{aligned}
$$

\section{B. Molecular model}

The potentials $U_{i j}\left(\psi_{1}, \psi_{2}, \mathbf{r}\right)$ participating in the mean field (4) may consist of the electrostatic and the dispersion parts. In the case of electrostatic interaction, let us expand the exponent function in Eq. (4) in Taylor series again up to the second term. In paper [1] only the linear part of the expression for the electrostatic dipole-dipole and dipolequadrupole mean field in Eq. (4) was derived on the assumption of a strong positional correlation between the nearest molecular dipoles located in neighboring layers. The square electrostatic term was taken directly from the model considered in [13]. Here we are going to derive both linear and square electrostatic terms in the framework of a similar molecular model. By contrast to paper [1], let us assume that the transverse dipole moment $\boldsymbol{\mu}_{i}$ is located in the one molecular 


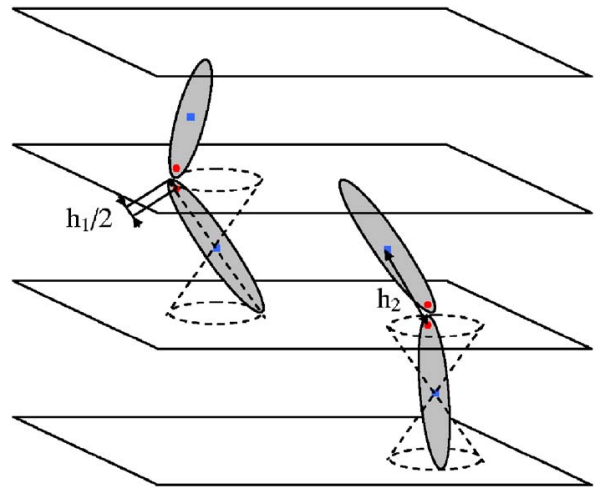

FIG. 1. (Color online) A snapshot of positionally correlated molecules in neighboring layers. Dipole moments are located in the molecular tails (one per molecule, see red circles), and quadrupole moments are located in the centers of molecules (blue squares). Orientations of the dipole and quadrupole moments are not shown to avoid the misleading conclusions.

tail (see Fig. 1), the uniaxial quadrupole moment [i.e., symmetrical with respect to the rotation of a molecule around its long axis: $q_{\alpha \beta}^{(i)} \equiv Q\left(n_{\alpha}^{(i)} n_{\beta}^{(i)}-1 / 3 \delta_{\alpha \beta}\right)$, where $n_{\alpha}^{(i)}$ and $n_{\beta}^{(i)}$ are the projections of the nematic director $\mathbf{n}_{i}$ in layer $i$ on some coordinate axes $\alpha$ and $\beta$ ] is still located in the center of the molecule, and orientations of molecules looking "up" and "down" have equal probabilities. In the following two sections $\mathrm{C}$ and $\mathrm{D}$ we are going to derive the expressions for the linear electrostatic mean field and for the dispersion mean field both modulated by the steric effect represented by the positional correlation function $g_{i j}\left(\mathbf{r}_{\perp}\right)$.

\section{Linear electrostatic interactions}

The dipole-dipole, dipole-quadrupole, and quadrupolequadrupole interactions are determined by the following expressions [1]:

$$
\begin{gathered}
U_{\mu \mu}^{i j} \equiv \mu_{\alpha}^{(i)} T_{\alpha \beta}\left(\mathbf{r}_{i j}\right) \mu_{\beta}^{(j)}, \\
U_{\mu q}^{i j} \equiv \mu_{\alpha}^{(i)} T_{\alpha \beta \gamma}\left(\mathbf{r}_{i j}\right) q_{\beta \gamma}^{(j)}, \\
U_{q q}^{i j} \equiv q_{\alpha \beta}^{(i)} T_{\alpha \beta \gamma \delta}\left(\mathbf{r}_{i j}\right) q_{\gamma \delta}^{(j)},
\end{gathered}
$$

where $T_{\alpha \beta}\left(\mathbf{r}_{i j}\right), T_{\alpha \beta \gamma}\left(\mathbf{r}_{i j}\right)$, and $T_{\alpha \beta \gamma \delta}\left(\mathbf{r}_{i j}\right)$ are the following coupling tensors:

$$
\begin{gathered}
T_{\alpha \beta}\left(\mathbf{r}_{i j}\right) \equiv-\nabla_{\alpha} \nabla_{\beta} \frac{1}{r_{i j}}, \\
T_{\alpha \beta \gamma}\left(\mathbf{r}_{i j}\right) \equiv \nabla_{\alpha} \nabla_{\beta} \nabla_{\gamma} \frac{1}{r_{i j}}, \\
T_{\alpha \beta \gamma \delta}\left(\mathbf{r}_{i j}\right) \equiv-\nabla_{\alpha} \nabla_{\beta} \nabla_{\gamma} \nabla_{\delta} \frac{1}{r_{i j}},
\end{gathered}
$$

and where $\mathbf{r}_{i j}$ is the vector connecting a multipole (dipole or quadrupole) of a molecule in layer $i$ with a multipole of a molecule in layer $j$. Then, in the framework of the present molecular model, the linear electrostatic term in the mean field (4) will be slightly different from that presented in [1]:

$$
\begin{aligned}
\frac{\rho^{2} \mu^{2}}{2 k_{B} T} U_{M F}^{\mathrm{el}(i)}(\mathbf{P}) \approx & -\boldsymbol{\mu}_{i} \hat{\mathbf{g}}_{0} \mathbf{P}_{i}+\boldsymbol{\mu}_{i} \hat{\mathbf{g}}_{1}\left(\mathbf{P}_{i+1}+\mathbf{P}_{i-1}\right) \\
& +\mu c_{f}\left(\rho \boldsymbol{\mu}_{i}+\mathbf{P}_{i}\right) \Delta \mathbf{n}_{i \pm 1},
\end{aligned}
$$

where $\mathbf{n}_{i}$ is the nematic director in layer $i, \Delta \mathbf{n}_{i \pm 1} \equiv \mathbf{n}_{i+1}$ $-\mathbf{n}_{i-1}, c_{f}$ is a flexoelectric constant:

$$
c_{f} \equiv-\frac{3}{2} Q \frac{\rho\left\langle g_{i, i \pm 1}\right\rangle \mu}{k_{B} T h_{2}^{4}},
$$

$\hat{\mathbf{g}}_{0}$ and $\hat{\mathbf{g}}_{1}$ are the following second rank tensors (see the Appendix):

$$
\begin{gathered}
\hat{\mathbf{g}}_{0} \equiv \frac{\rho\left\langle g_{i, i}\right\rangle \mu^{2}}{8 k_{B} T d^{3}}\left[\delta_{\alpha \beta}\left(1-\frac{3}{2} \theta^{2}\right)+\frac{3}{2} w_{\alpha}^{(i)} w_{\beta}^{(i)} \theta^{2}\right], \\
\hat{\mathbf{g}}_{1} \equiv \frac{\rho\left\langle g_{i, i \pm 1}\right\rangle \mu^{2}}{4 k_{B} T h_{1}^{3} \cos ^{3} \theta}\left(\delta_{\alpha \beta}-3 k_{\alpha} k_{\beta}\right),
\end{gathered}
$$

and where $d$ is the breadth of a molecule, $\theta$ is the tilt angle (which is assumed to be small), $\mathbf{w}_{i}$ is the unit vector perpendicular to the tilt plane in layer $i, h_{1} \cos \theta$ and $h_{2} \approx \ell / 2$ are the distances between the two nearest dipole moments and between the nearest dipole and quadrupole moments in the neighboring layers, respectively (see Fig. 1), the averages $\left\langle g_{i, j}\right\rangle$ are defined as follows:

$$
\left\langle g_{i, j}\right\rangle \equiv \int g_{i, j}\left(\mathbf{r}_{\perp}\right) d^{2} \mathbf{r}_{\perp}
$$

and $\mathbf{P}_{i}$ is polarization of layer $i$ :

$$
\mathbf{P}_{i}=\rho \int_{0}^{2 \pi} \boldsymbol{\mu}_{i} f_{i}(\psi) d \psi .
$$

For simplicity in paper [1] we considered both molecular dipole and quadrupole moments located in the centers of molecules. It is well-known, however, that intermediate phases and even $\mathrm{Sm}-C_{A}^{*}$ are usually observed in the systems of molecules with terminal dipoles. This seems to be reasonable because otherwise molecules in neighboring layers have weak positional correlation, and it may be shown that without this correlation the average dipole-dipole and dipolequadrupole interaction of molecules in neighboring layers is equal to zero. Thus it is important to consider terminal dipoles in the molecular model. At the same time, quadrupole moments are usually associated with rigid molecular cores, and thus it is reasonable to place quadrupole moment in the center of a molecule. In this case the vector connecting quadrupole moment of the first molecule and dipole moment of the second one is not parallel to vector $\mathbf{k}$ anymore, but almost parallel to the nematic director of the first molecule, and thus the multiplier (n.k) in the third term of Eq. (9) is removed by contrast to paper [1].

\section{Dispersion interactions}

The polar terms in the mean field may also arise from nonpolar interactions (for example, dispersion attraction 


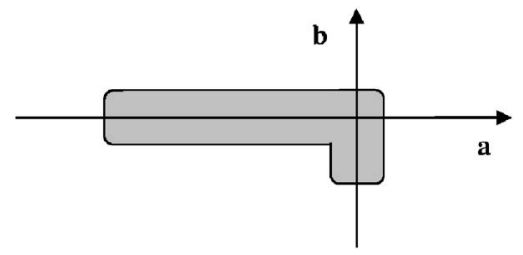

FIG. 2. An example of molecule polar in two directions.

modulated by polar molecular shape). In general, any interactions may be approximated by spherical invariants $T^{\ell L \lambda}\left(\mathbf{x}_{i}, \mathbf{r}, \mathbf{y}_{j}\right)$, where $\mathbf{x}$ and $\mathbf{y}$ may be either along the long molecular axes $\mathbf{a}_{i}$ and $\mathbf{a}_{j}$, or one of them may be along the short molecular axis ( $\mathbf{b}_{i}$ or $\mathbf{c}_{i}$ instead of $\mathbf{a}_{i}$, or $\mathbf{b}_{j}$ or $\mathbf{c}_{j}$ instead of $\mathbf{a}_{j}$ ), or both may be along the short molecular axes instead of the corresponding long axes. The basic nonpolar spherical invariants are presented, for example, in [14]. Suppose that in addition molecules have polar shape in two directions (i.e., for example, vector a cannot be replaced with $-\mathbf{a}$, and vector $\mathbf{b}$ cannot be replaced with -b) (see Fig. 2). By defining vector $\mathbf{c} \equiv[\mathbf{a} \times \mathbf{b}]$ for every molecule, one notes that the following simplest polar term will arise in the dispersion interaction between two molecules located in layers $i$ and $j$ :

$$
\begin{aligned}
T^{221}\left(\mathbf{n}_{i}, \mathbf{r}, \mathbf{c}_{j}\right)+T^{221}\left(\mathbf{n}_{j}, \mathbf{r}, \mathbf{c}_{i}\right) \equiv & \left(\mathbf{n}_{i} \cdot \mathbf{r}\right)\left(\mathbf{c}_{j} \cdot\left[\mathbf{n}_{i} \times \mathbf{r}\right]\right) \\
& +\left(\mathbf{n}_{j} \cdot \mathbf{r}\right)\left(\mathbf{c}_{i} \cdot\left[\mathbf{n}_{j} \times \mathbf{r}\right]\right) .
\end{aligned}
$$

Suppose that interaction of kind (14) within the same layer only is essentially present. Here we are not interested in the nonpolar ordering of the short molecular axes and polar ordering of the long molecular axes, and thus will assume that the corresponding terms proportional to the even powers of $\mathbf{c}_{i}$ and $\mathbf{c}_{j}$ and odd powers of $\mathbf{n}_{i}$ and $\mathbf{n}_{j}$ are already averaged with respect to the orientations of these vectors. Then the nonpolar spherical invariants may be written in the manner of paper [13], and one obtains the following expression for the dispersion mean field in the case of positionally correlated molecules in neighboring layers:

$$
\begin{aligned}
U_{M F}^{\mathrm{disp}(i)}= & v_{0}+v_{1}\left[P_{2}\left(\mathbf{n}_{i} \cdot \mathbf{n}_{i-1}\right)+P_{2}\left(\mathbf{n}_{i} \cdot \mathbf{n}_{i+1}\right)\right]+v_{2} \cos ^{2} \theta \\
& +v_{3} \cos ^{2} \theta\left[\left(\mathbf{n}_{i} \cdot \mathbf{n}_{i-1}\right)+\left(\mathbf{n}_{i} \cdot \mathbf{n}_{i+1}\right)\right]+v_{4} \cos ^{4} \theta \\
& +v_{5}\left\{\left(\mathbf{n}_{i} \cdot \mathbf{n}_{i-1}\right)\left(\mathbf{k} \cdot\left[\mathbf{n}_{i} \times \mathbf{n}_{i-1}\right]\right)\right. \\
& \left.+\left(\mathbf{n}_{i} \cdot \mathbf{n}_{i+1}\right)\left(\mathbf{k} \cdot\left[\mathbf{n}_{i} \times \mathbf{n}_{i+1}\right]\right)\right\}+v_{6}\left(\mathbf{c}_{i}+\left\langle\mathbf{c}_{i}\right\rangle\right) \xi_{i},
\end{aligned}
$$

where we assume that the tilt angle $\theta$ is the same in every layer, and thus $\left(\mathbf{n}_{i-1} \cdot \mathbf{k}\right)=\left(\mathbf{n}_{i+1} \cdot \mathbf{k}\right)=\left(\mathbf{n}_{i} \cdot \mathbf{k}\right)=\cos \theta$. Zeroth, second, and fourth terms in Eq. (15) contain both nonpolar dispersion interactions of molecules within the same layer and in the neighboring layers, whereas the first, third, and fifth terms are determined by the interactions of molecules in the neighboring layers only, and the additional sixth term corresponds to polar spherical invariant (14), where $\xi_{i}$ $\equiv\left(\mathbf{n}_{i} \cdot \mathbf{k}\right)\left[\mathbf{n}_{i} \times \mathbf{k}\right]=\left|\xi_{i}\right| \mathbf{w}_{i}$.

\section{E. Polarization-dependent free energy}

The electrostatic contribution (9) and the dispersion contribution (15) to the mean field must be now substituted into the free energy (6). The fifth term in Eq. (15) arises if molecules are chiral. At the same time, molecules must not be chiral to produce the nonzero sixth term in Eq. (15), because vectors $\mathbf{c}$ for every molecule were defined artificially, and nothing special corresponds to these directions so far. Nevertheless, the sixth term itself will vanish after the averaging procedure in Eq. (6), because

$$
\int_{0}^{2 \pi} f(\psi) d \psi=1
$$

and thus, the first and the last terms in Eq. (6) corresponding to the sixth term in Eq. (15) will compensate each other. At the same time, if the transverse dipole moment $\boldsymbol{\mu}$ has a nonzero projection on vector $\mathbf{c}$, the second term in Eq. (6) will produce the coupling between polar electrostatic and polar dispersion interactions, and an additional chiral term in the free energy depending on polarization will arise. For simplicity, let us assume that $\boldsymbol{\mu}$ is parallel to c. Collecting the corresponding terms, one obtains the following expression for the polarization dependent free energy of layer $i$ :

$$
\begin{aligned}
\frac{\rho \mu^{2}}{k_{B} T} F_{\mathbf{p}}^{(i)}= & \rho^{2} \mu^{2} \mathbf{M}_{i}^{2}+\mathbf{P}_{i} \hat{\mathbf{g}}_{0}\left(\hat{\mathbf{I}}-\hat{\mathbf{g}}_{0}\right) \mathbf{P}_{i}-\mathbf{P}_{i} \hat{\mathbf{g}}_{1}\left(\hat{\mathbf{I}}-2 \hat{\mathbf{g}}_{0}\right) \\
& \times\left(\mathbf{P}_{i-1}+\mathbf{P}_{i+1}\right)-\left(\mathbf{P}_{i-1}+\mathbf{P}_{i+1}\right) \hat{\mathbf{g}}_{1}^{2}\left(\mathbf{P}_{i-1}+\mathbf{P}_{i+1}\right) \\
& +2 \rho \mu \mathbf{M}_{i}\left[\hat{\mathbf{g}}_{0} \mathbf{P}_{i}-\hat{\mathbf{g}}_{1}\left(\mathbf{P}_{i-1}+\mathbf{P}_{i+1}\right)-\rho \mu \mathbf{M}_{i}\right],
\end{aligned}
$$

where $\hat{\mathbf{I}} \equiv \delta_{\alpha \beta}$ and vector $\mathbf{M}_{i}$ plays a role of the complex order parameter:

$$
\mathbf{M}_{i} \equiv c_{p}\left[\mathbf{n}_{i} \times \mathbf{k}\right]+c_{f}\left[\mathbf{n}_{i} \times\left[\Delta \mathbf{n}_{i \pm 1} \times \mathbf{n}_{i}\right]\right],
$$

where $c_{f}$ is determined by Eq. (10), and $c_{p}$ (designated as $c_{s}$ in [1]) is determined by the following expression:

$$
c_{p} \equiv \frac{\rho\left\langle g_{i, i}\right\rangle v_{6} \cos \theta}{k_{B} T} .
$$

The first term in Eq. (18) is known as the "piezoelectric" term. In our model (that looks complete) it arises because of the coupling between the polar in two directions dispersion interaction [the sixth term in Eq. (15)] and the dipole-dipole interaction [the first two terms in Eq. (9)]. One notes that a molecule with polar shape in two directions $\mathbf{a}$ and $\mathbf{b}$ (see Fig. 2 ) and having dipole moment with nonzero projection on vector $\mathbf{c} \equiv[\mathbf{a} \times \mathbf{b}]$ is polar already in three directions not lying in the same plane. Since these directions in addition cannot be replaced with each other, this molecule is chiral. This chirality, however, emerges only as a combination of the dispersion and the dipole-dipole interactions, so let us call it "combined" chirality in contrast to the "conventional" chirality arising solely from the dispersion interaction modulated by the chiral molecular shape [the fifth term in Eq. (15)].

The second term in Eq. (18) is actually a "flexoelectric" term. Indeed, double vector product $\left[\mathbf{n}_{i} \times\left[\Delta \mathbf{n}_{i \pm 1} \times \mathbf{n}_{i}\right]\right]$ may be expanded as a sum of the two scalar products multiplied by a vector, and one obtains the same two flexoelectric terms 
$\Delta \mathbf{n}_{i \pm 1}$ and $-\mathbf{n}_{i}\left(\mathbf{n}_{i} \cdot \Delta \mathbf{n}_{i \pm 1}\right)$ as in [1]. Presentation (18), however, is more convenient, because it is easily seen from this presentation that the flexoelectric term is always perpendicular to $\mathbf{n}_{i}$, but (by contrast to the piezoelectric term) may have a contribution parallel to the local tilt plane if $\Delta \mathbf{n}_{i \pm 1}$ is not perpendicular to $\mathbf{n}_{i}$. One can easily check that in the case of the biaxial subphases these two vectors are indeed not perpendicular to each other, and thus, the flexoelectric term must not be parallel to the piezoelectric one. Moreover, from the second part $-\mathbf{n}_{i}\left(\mathbf{n}_{i} \cdot \Delta \mathbf{n}_{i \pm 1}\right)$ it is seen that the whole flexoelectric term must have a vertical contribution (perpendicular to the smectic layer plane), since the first part $\Delta \mathbf{n}_{i \pm 1}$ does not have a vertical contribution in the case of the same tilt angle in every layer. The vertical flexoelectric contribution is proportional to the additional power of the small tilt angle in comparison with the horizontal one. In paper [1] we neglected this term for simplicity, only noting that it produces relatively weak polarization perpendicular to the layer plane. Now we are going to take the temperature dependence of the tilt angle into account and, thus, we keep the vertical contribution in Eq. (18). In our model the whole flexoelectric contribution arises because of the coupling between the dipoledipole and the dipole-quadrupole interactions. One can see that in our model the symmetries of the piezoelectric and the flexoelectric terms are intimately related with their origins.

Finally, the coupling between the two dipole-dipole interactions within the same smectic layer and in the neighboring layers, as well as the first powers of the dipole-dipole interactions, produce the other terms (independent of $\mathbf{M}_{i}$ ) in the free energy (17). The first power of the dipole-quadrupole interaction disappears from the free energy (on average) due to the symmetry, and the first powers of the dispersion and quadrupole-quadrupole interactions contribute to the polarization-independent free energy, which is considered in the next section F. Minimizing the total free energy $F_{\mathbf{p}}$ [that is a sum of $F_{\mathbf{p}}^{(i)}$ ] with respect to polarization vectors one obtains the following set of equations for polarizations $\mathbf{P}_{i}$ :

$$
\begin{aligned}
& \hat{\mathbf{g}}_{0}\left(\hat{\mathbf{I}}-\hat{\mathbf{g}}_{0}\right) \mathbf{P}_{i}-\hat{\mathbf{g}}_{1}\left(\hat{\mathbf{I}}-2 \hat{\mathbf{g}}_{0}\right)\left(\mathbf{P}_{i-1}+\mathbf{P}_{i+1}\right)-\hat{\mathbf{g}}_{1}^{2}\left(2 \mathbf{P}_{i}+\mathbf{P}_{i-2}+\mathbf{P}_{i+2}\right) \\
& \quad+\rho \mu \hat{\mathbf{g}}_{0} \mathbf{M}_{i}-\rho \mu \hat{\mathbf{g}}_{1}\left(\mathbf{M}_{i-1}+\mathbf{M}_{i+1}\right)=0 .
\end{aligned}
$$

Looking at Eq. (20) one may think that there is some correlation between polarization vectors in the next-neighboring layers. Nevertheless, the number of correlating layers reduces to only neighboring ones. Indeed, substituting equation

$$
\left(\hat{\mathbf{I}}-\hat{\mathbf{g}}_{0}\right) \mathbf{P}_{i}+\hat{\mathbf{g}}_{1}\left(\mathbf{P}_{i-1}+\mathbf{P}_{i+1}\right)=-\rho \mu \mathbf{M}_{i}
$$

into Eq. (20), one obtains again Eq. (21). Thus, Eqs. (20) and (21) are equivalent. Substituting Eqs. (20) and (21) into Eq. (17), one can essentially simplify the expression for the polarization dependent free energy of layer $i$ :

$$
\frac{\mu}{k_{B} T} F_{\mathbf{p}}^{(i)}=\mathbf{M}_{i} \cdot \mathbf{P}_{i}+\rho \mu \mathbf{M}_{i}^{2},
$$

where the order parameters $\mathbf{M}_{i}$ are given by Eq. (18), and a set of polarization vectors $\mathbf{P}_{i}$ is still determined by Eq. (21). In contrast to paper [1], a direct coupling between the order parameters within the same smectic layer [the second term in
Eq. (22)] arises after a consideration of the total free energy. One notes from Eqs. (11) and (21) that anisotropy of tensors $\hat{\mathbf{g}}_{0}$ and $\hat{\mathbf{g}}_{1}$ produces additional polarization along vectors $\mathbf{w}_{i}$ and $\mathbf{k}$, respectively. Using index $\alpha_{i}=\perp, w_{i}$ or $m_{i}$ for enumeration of the projections of different vectors on axes $\mathbf{k}, \mathbf{w}_{i}$, or $\mathbf{m}_{i} \equiv\left[\mathbf{k} \times \mathbf{w}_{i}\right]$, respectively, one can rewrite Eqs. (21) in the following form:

$$
P_{i}^{\left(\alpha_{i}\right)}+g_{\alpha}\left[P_{i-1}^{\left(\alpha_{i}\right)}+P_{i+1}^{\left(\alpha_{i}\right)}\right]=-\rho \mu \chi_{\alpha} M_{i}^{\left(\alpha_{i}\right)},
$$

where in correspondence with Eq. (11)

$$
\begin{gathered}
\chi_{w}=\left(1-g_{0}\right)^{-1}, \\
\chi_{m}=\chi_{\perp} \approx \chi_{w}\left(1-\frac{3}{2} \chi_{w} g_{0} \theta^{2}\right), \\
g_{w}=g_{1} \chi_{w}, \quad g_{m}=g_{1} \chi_{m}, \quad g_{\perp}=-2 g_{1} \chi_{\perp},
\end{gathered}
$$

and where

$$
g_{0} \equiv \frac{\rho\left\langle g_{i, i}\right\rangle \mu^{2}}{8 k_{B} T d^{3}}, \quad g_{1} \equiv \frac{\rho\left\langle g_{i, i \pm 1}\right\rangle \mu^{2}}{4 k_{B} T h_{1}^{3} \cos ^{3} \theta} .
$$

One notes from Eqs. (24) that anisotropy of the dielectric susceptibility is proportional to the second power of the small tilt angle $\theta$. Set of equations (23) may be solved with respect to polarizations in any particular case of the smectic structure with periodicity of $t$ layers. Polarization vectors may be subsequently eliminated from the free energy (22) that may be rewritten in terms of the effective coupling between the order parameters $\mathbf{M}_{i}$ :

$$
\frac{F_{\mathbf{p}}}{\rho k_{B} T}=-\frac{N}{t} \sum_{i=0}^{t-1} \sum_{k=0}^{t-1} \sum_{\alpha_{i}=1}^{3}\left[\chi_{\alpha} f_{k}^{(\alpha)}-\delta_{k 0}\right] M_{i}^{\left(\alpha_{i}\right)} M_{i+k}^{\left(\alpha_{i}\right)},
$$

where coefficients $f_{k}^{(\alpha)}=f_{|j-i|}^{(\alpha)} \equiv f_{i j}^{(\alpha)}$ are the elements of the inverse matrix that is presented in the left part of Eq. (23). In other words, if we have the matrix with elements $a_{i i}^{(\alpha)} \equiv 1$, $a_{i, i \pm 1}^{(\alpha)}=a_{0, t-1}^{(\alpha)}=a_{t-1,0}^{(\alpha)} \equiv g_{\alpha}$ and the remaining elements equal to zero, then $\left\|f_{i j}^{(\alpha)}\right\| \equiv\left\|a_{i j}^{(\alpha)}\right\|^{-1}$. One can check that coefficients $f_{k}^{(\alpha)}$ decay as $\left(-g_{\alpha}\right)^{k}$ for $k=0 \ldots \pm([t / 2]+1)$, where $g_{\alpha}<1$ and $[t / 2]$ is the integer part of $t / 2$, although the exact expressions for this decay depend on the period $t$.

\section{F. Polarization-independent free energy}

Finally let us derive the expression for that part of the free energy, which is independent of polarization. The dispersion part of this expression is produced by the first six terms (from zeroth to fifth) in the dispersion mean field (15), whereas the electrostatic part derives from the quadrupolequadrupole interaction and from the second term in the expansion of the exponent in Eq. (4) where the electrostatic interactions (7) must be substituted. Here we are going to take also into account the quadrupole-quadrupole interaction between the molecules located in the next-neighboring layers, because the molecular quadrupole moment may be large. Both nonpolar interactions (the dispersion and the electrostatic ones) contribute only to the first term of Eq. (6). For 
simplicity let us assume that positional correlation of molecules in the neighboring layers is strong, and thus, $\rho\left\langle g_{i, i \pm 1}\right\rangle \approx 1$. Then collecting the corresponding terms and adding the polarization-dependent contribution (27), one obtains the following expression for the free energy of the intermediate phase with periodicity of $t$ layers:

$$
\begin{aligned}
\frac{t\left[F-F_{0}(\theta)\right]}{\rho N k_{B} T}= & \left(\frac{3 v_{1}}{2 k_{B} T}-g_{1}^{2}+4 c_{f}^{2}\right) \sum_{i=0}^{t-1}\left(\mathbf{n}_{i} \cdot \mathbf{n}_{i+1}\right)^{2} \\
& +\left(\frac{v_{3}}{k_{B} T}+6 g_{1}^{2}\right) \cos ^{2} \theta \sum_{i=0}^{t-1}\left(\mathbf{n}_{i} \cdot \mathbf{n}_{i+1}\right) \\
& +\frac{v_{5}}{k_{B} T} \sum_{i=0}^{t-1}\left(\mathbf{n}_{i} \cdot \mathbf{n}_{i+1}\right)\left(\mathbf{k} \cdot\left[\mathbf{n}_{i} \times \mathbf{n}_{i+1}\right]\right) \\
& +\frac{10}{3}\left(\frac{h_{2}}{h_{1}}\right)^{3}\left(\frac{h_{2}}{h_{3}}\right)^{5} \frac{c_{f}^{2}}{g_{1} \cos \theta} \sum_{i=0}^{t-1}\left(\mathbf{n}_{i} \cdot \mathbf{n}_{i+2}\right) \\
& -\sum_{i=0}^{t-1} \sum_{k=0}^{t-1} \sum_{\alpha_{i}=1}^{3}\left[\chi_{\alpha} f_{k}^{(\alpha)}-\delta_{k 0}\right] M_{i}^{\left(\alpha_{i}\right)} M_{i+k}^{\left(\alpha_{i}\right)},
\end{aligned}
$$

where the first three terms represent the interaction between molecules in neighboring layers [where the terms proportional to $v_{1}$ and $v_{3}$ represent the nonchiral dispersion interaction, the term proportional to $v_{5}$ represents the chiral dispersion interaction, the terms proportional to $g_{1}^{2}$ represent the square dipole-dipole interaction, and the term proportional to $c_{f}^{2}$ represents the square dipole-quadrupole interaction (only transverse molecular dipoles participate in both cases)], the fourth term represents the quadrupole-quadrupole interaction in the next-neighboring layers [where $h_{3} \approx 2(\ell+d)$ is the distance between the correlated quadrupoles in the nextneighboring layers], and the fifth term is the effective longrange coupling arising from the polarization-dependent free energy. For simplicity, the electrostatic interactions produced by molecular longitudinal dipoles, as well as quadrupolequadrupole and higher multipole interactions between molecules located in the neighboring layers, are incorporated into the dispersion interaction (into the terms with coefficients $v_{1}$ and $v_{3}$, which are known as quadrupolar and dipolar terms, respectively).

\section{G. Some notes about the biaxial nonpolar ordering}

In expression (28) for the free energy the biaxial nonpolar ordering was neglected. One notes that in the general case the following average procedure is valid for the product of two projections of the transverse dipole moment $\boldsymbol{\mu}_{i}$ :

$$
\begin{aligned}
\frac{1}{2 \pi} \int_{0}^{2 \pi} \mu_{i}^{(\alpha)} \mu_{i}^{(\beta)} f_{i}(\psi) d \psi= & \sigma P_{i}^{(\alpha)} P_{i}^{(\beta)} \mu^{2} / P^{2} \\
& +\frac{1}{2}(1-\sigma) \mu^{2}\left[\delta_{\alpha \beta}-n_{i}^{(\alpha)} n_{i}^{(\beta)}\right],
\end{aligned}
$$

where indexes $\alpha$ and $\beta$ denote the projections of the corresponding vectors on the axes of the coordinate system, and where $\sigma \equiv\langle\cos 2 \psi\rangle$ is the biaxial (nonpolar) order parameter. In Eq. (28) it was assumed to be small, and all terms proportional to $\sigma$ were neglected. This supposition seems to be quite reasonable far from the $\mathrm{Sm}-C^{*}-\mathrm{Sm}-A^{*}$ transition point, where the polar order parameter $P=\rho \mu\langle\cos \psi\rangle$ (called "polarization") is known to play the more important role. At the same time, in the vicinity of the $\mathrm{Sm}-C^{*}-\mathrm{Sm}-A^{*}$ transition point, the polarization becomes very small (and disappears completely in the transition point), whereas the nonpolar biaxial order parameter $\sigma$ remains almost the same (similarly to the nematic order parameter $S$ ). In this case it appears to be the only parameter determining the helical rotation, and thus it must be taken into account. This will be done in Sec. IV.

\section{PERTURBATION THEORY FOR THE INTERMEDIATE PHASES EXHIBITING HELICAL ROTATION}

\section{A. Formulation neglecting the nonpolar biaxial ordering}

First let us simplify Eq. (27) for the polarizationdependent part of the free energy taking into account only terms proportional to the power of the small parameter $\sin \theta$ not higher than four. In Sec. II it was noticed [see Eq. (18) and discussion thereafter] that order parameter $\mathbf{M}_{i}$ consists of the main horizontal (parallel to the smectic layer plane) contribution (let us call it $\mathbf{M}_{i}^{\|}$) which is proportional to the powers of $\sin \theta$ not higher than one, and of the small addition (let us call it $\left.\Delta \mathbf{M}_{i}\right)$ which is proportional to the higher powers of $\sin \theta$ and has both horizontal and vertical projections:

$$
\begin{aligned}
& \mathbf{M}_{i}^{\|}=c_{p}\left[\mathbf{n}_{i} \times \mathbf{k}\right]+c_{f} \Delta \mathbf{n}_{i \pm 1}, \\
& \Delta \mathbf{M}_{i}=-c_{f}\left[\mathbf{n}_{i}\left(\mathbf{n}_{i} \cdot \Delta \mathbf{n}_{i \pm 1}\right)\right] .
\end{aligned}
$$

In our approximation the anisotropy of the dielectric susceptibility [which is proportional to $\theta^{2}$ itself, see Eqs. (24) and (25)] may be taken into account only for the coupling of the main horizontal contributions, and one obtains:

$$
\begin{aligned}
\frac{t F_{\mathbf{p}}}{\rho N k_{B} T} \approx & -\sum_{i=0}^{t-1} \sum_{k=0}^{t-1}\left[\chi_{m} f_{k}^{(m)}-\delta_{k 0}\right] \mathbf{M}_{i}^{\|} \mathbf{M}_{i+k}^{\|} \\
& -\sum_{i=0}^{t-1} \sum_{k=0}^{t-1}\left[\chi_{w} f_{k}^{(w)}-\chi_{m} f_{k}^{(m)}\right] M_{i}^{\left(w_{i}\right)} M_{i+k}^{\left(w_{i}\right)} \\
& -\sum_{i=0}^{t-1} \sum_{k=0}^{t-1}\left[\chi_{w} f_{k}^{(w)}-\delta_{k 0}\right]\left(\mathbf{M}_{i}^{\|} \Delta \mathbf{M}_{i+k}+\Delta \mathbf{M}_{i} \mathbf{M}_{i+k}^{\|}\right) \\
& -\sum_{i=0}^{t-1} \sum_{k=0}^{t-1}\left[\chi_{\perp} f_{k}^{\perp}-\delta_{k 0}\right] \Delta \mathbf{M}_{i} \Delta \mathbf{M}_{i+k} .
\end{aligned}
$$

In contrast to paper [1] let us assume that the structure does not repeat itself completely in a period $t$, but exhibits a small additional rotation $\Delta \varphi$ per layer. At first glance, in the general case (including biaxial phase) we must write different additional rotation angles $\Delta \varphi_{i, i+1}(i=0, \ldots, t-1)$ for various layers. Let us show that only one of these angles is independent. If we suppose that the angles $\Delta \varphi_{i, i+1}$ are different, the total angles of the azimuthal rotation from layer to layer will 
be $\varphi_{i, i+1}+\Delta \varphi_{i, i+1}(i=0, \ldots, t-1)$. We remind that angles $\varphi_{i, i+1}$ were chosen to satisfy the constraint $\Sigma_{i=0}^{t-1} \varphi_{i, i+1}=2 \pi$, i.e., they correspond to the phase with periodicity of $t$ layers without additional helical rotation, and the angles $\Delta \varphi_{i, i+1}$ describe the additional helical rotation. Now let us transfer from $\varphi_{i, i+1}$ to $\varphi_{i, i+1}^{\text {new }}$ according to the following rule: $\varphi_{i, i+1}^{\text {new }} \equiv \varphi_{i, i+1}+\Delta \varphi_{i, i+1}$ $-\Delta \varphi$, where $\Delta \varphi \equiv 1 / t \Sigma_{i=0}^{t-1} \Delta \varphi_{i, i+1}$. One notes that the previous constraint is still valid: $\Sigma_{i=0}^{t-1} \varphi_{i, i+1}^{\text {new }}=2 \pi$, but the total set of the azimuthal rotation angles from layer to layer may now be written with the help of only one helical rotation angle: $\varphi_{i, i+1}^{\text {new }}+\Delta \varphi(i=0, \ldots, t-1)$. Since there is no difference, which angles to choose for minimization, let us choose the angles $\varphi_{i, i+1}^{\text {new }}$ (index "new" will be omitted for the following consideration).

At first glance we now need to express the nematic director $\mathbf{n}_{i}$ and the difference $\Delta \mathbf{n}_{i \pm 1}$ participating in Eq. (30) in terms of the tilt angle $\theta$ and the azimuthal angles $\varphi_{i}+i \Delta \varphi$, and then simply to substitute Eq. (30) into Eq. (31). We cannot do this, however, because parameter $\mathbf{M}_{i}$ presented in this form is not periodical anymore, and Eq. (31), obtained itself in supposition that parameter $\mathbf{M}_{i}$ has a period of $t$ smectic layers, is not valid in this case. At the same time, we can use a perturbation scheme which assumes the helical rotation $\Delta \varphi$ to be small. For example, we can fix the nematic director $\mathbf{n}_{i}$ in every layer as it was in the absence of helical rotation, and modify only the difference $\Delta \mathbf{n}_{i \pm 1}$ :

$$
\begin{gathered}
\mathbf{n}_{i}=\sin \theta\left(\mathbf{x} \cos \varphi_{i}+\mathbf{y} \sin \varphi_{i}\right)+\mathbf{k} \cos \theta, \\
\Delta \mathbf{n}_{i \pm 1} / \sin \theta=\mathbf{x}\left[\cos \left(\varphi_{i+1}+\Delta \varphi\right)-\cos \left(\varphi_{i-1}-\Delta \varphi\right)\right] \\
+\mathbf{y}\left[\sin \left(\varphi_{i+1}+\Delta \varphi\right)-\sin \left(\varphi_{i-1}-\Delta \varphi\right)\right],
\end{gathered}
$$

where $\mathbf{x}$ and $\mathbf{y}$ are some orthogonal unit vectors in the layer plane, and $\varphi_{i+t}=\varphi_{i}$. In this case the order parameters $\mathbf{M}_{i}$ exhibit a small variation similar to the helical rotation, but still remain periodical with period $t$, and we can still use Eq. (31) for the polarization-dependent free energy together with the coefficients $f_{k}^{(\alpha)}$ obtained on the assumption of the absence of helical rotation. Let us express the scalar products of the order parameters participating in Eq. (31) in terms of the tilt and the azimuthal angles using Eqs. (30) and (32):

$$
\begin{aligned}
\mathbf{M}_{i}^{\|} \mathbf{M}_{j}^{\|}= & \sin ^{2} \theta\left\{c_{p}^{2} \cos \varphi_{i j}+c_{f}^{2}\left[\cos \varphi_{i+1, j+1}+\cos \varphi_{i-1, j-1}\right.\right. \\
& \left.-\cos \left(\varphi_{i-1, j+1}+2 \Delta \varphi\right)-\cos \left(\varphi_{i+1, j-1}-2 \Delta \varphi\right)\right] \\
& -c_{p} c_{f}\left[\sin \left(\varphi_{i, j+1}+\Delta \varphi\right)-\sin \left(\varphi_{i, j-1}-\Delta \varphi\right)\right. \\
& \left.\left.+\sin \left(\varphi_{i-1, j}+\Delta \varphi\right)-\sin \left(\varphi_{i+1, j}-\Delta \varphi\right)\right]\right\},
\end{aligned}
$$

$$
\begin{aligned}
\Delta \mathbf{M}_{i} \mathbf{M}_{j}^{\|}= & -c_{f} \sin ^{4} \theta\left[\cos \left(\varphi_{i, i+1}+\Delta \varphi\right)-\cos \left(\varphi_{i-1, i}+\Delta \varphi\right)\right] \\
& \times\left\{c_{p} \sin \varphi_{i j}+c_{f}\left[\cos \left(\varphi_{i, j+1}+\Delta i\right)\right.\right. \\
& \left.\left.-\cos \left(\varphi_{i, j-1}-\Delta \varphi\right)\right]\right\},
\end{aligned}
$$

$$
\begin{aligned}
\Delta \mathbf{M}_{i} \Delta \mathbf{M}_{j}= & c_{f}^{2} \sin ^{4} \theta \cos ^{2} \theta\left[\cos \left(\varphi_{i, i+1}+\Delta \varphi\right)-\cos \left(\varphi_{i-1, i}\right.\right. \\
& +\Delta \varphi)]\left[\cos \left(\varphi_{j, j+1}+\Delta \varphi\right)-\cos \left(\varphi_{j-1, j}+\Delta \varphi\right)\right],
\end{aligned}
$$

where $\varphi_{i j}$ are the azimuthal differences between the director orientations in layers $i$ and $j$. In the expression for $\Delta \mathbf{M}_{i} \Delta \mathbf{M}_{j}$ we have again neglected the terms proportional to the powers of $\sin \theta$ higher than four. Defining, for example, $\mathbf{x}=\mathbf{m}_{i}$ and $\mathbf{y}=-\mathbf{w}_{i}$, where $\mathbf{w}_{i} \equiv \boldsymbol{\xi}_{i} /\left|\boldsymbol{\xi}_{i}\right|$ is the unit vector perpendicular to the tilt plane, and $\mathbf{m}_{i} \equiv\left[\mathbf{k} \times \mathbf{w}_{i}\right]$ (see Sec. II), one can also write:

$$
\begin{aligned}
M_{i}^{\left(w_{i}\right)} M_{j}^{\left(w_{i}\right)}= & \sin ^{2} \theta\left\{c_{p}-c_{f}\left[\sin \left(\varphi_{i, i+1}+\Delta \varphi\right)+\sin \left(\varphi_{i-1, i}\right.\right.\right. \\
& +\Delta \varphi)]\}\left\{c_{p} \cos \varphi_{i j}-c_{f}\left[\sin \left(\varphi_{i, j+1}+\Delta \varphi\right)\right.\right. \\
& \left.\left.-\sin \left(\varphi_{i, j-1}-\Delta \varphi\right)\right]\right\} .
\end{aligned}
$$

Then substituting Eqs. (33) and (34) into Eq. (31) and adding the other terms from Eq. (28), one obtains the following expression for the free energy in terms of the angles $\theta, \varphi i j$, and $\Delta \varphi$ :

$$
\frac{t\left[F-F_{0}(\theta)\right]}{\rho N k_{B} T}=\tilde{F}+\tilde{F}_{\mathbf{p}}+\Delta \tilde{F}_{\mathbf{p}}^{(1)}+\Delta \tilde{F}_{\mathbf{p}}^{(2)},
$$

where the first term $\widetilde{F}$ arises from the nonpolar interaction between molecules [the first four terms in Eq. (28)]:

$$
\begin{aligned}
\widetilde{F}= & \sin ^{2} \theta \sum_{i=0}^{t-1}\left\{b \sin ^{2} \theta \cos ^{2}\left(\varphi_{i, i+1}+\Delta \varphi\right)+\cos ^{2} \theta\left[a \operatorname { c o s } \left(\varphi_{i, i+1}\right.\right.\right. \\
& \left.+\Delta \varphi)+a_{1} \cos \left(\varphi_{i, i+2}+2 \Delta \varphi\right)+c_{1} \sin \left(\varphi_{i, i+1}+\Delta \varphi\right)\right] \\
& \left.\left.+\frac{1}{2} c_{2} \sin ^{2} \theta \sin \left(2 \varphi_{i, i+1}+2 \Delta \varphi\right)\right]\right\},
\end{aligned}
$$

where

$$
\begin{gathered}
a \equiv \frac{3 v_{1}+v_{3}}{k_{B} T}+4 g_{1}^{2}+8 c_{f}^{2}, \\
a_{1} \equiv \frac{10}{3}\left(\frac{h_{2}}{h_{1}}\right)^{3}\left(\frac{h_{2}}{h_{3}}\right)^{5} \frac{c_{f}^{2}}{g_{1} \cos ^{3} \theta}, \\
b \equiv \frac{3 v_{1}}{2 k_{B} T}-g_{1}^{2}+4 c_{f}^{2}, \quad c_{1}=c_{2} \equiv \frac{v_{5}}{k_{B} T} .
\end{gathered}
$$

It was discussed, for example, in [13] that both $v_{1}$ and $3 v_{1}$ $+v_{3}$ are negative. At the same time, $g_{1}^{2}$ and $c_{f}^{2}$ are positive. One notes from Eqs. (10) and (26) that $c_{f}$ and especially $g_{1}$ grow with the increasing dipole moment $\mu$, and thus, in the absence of polarization effects and chirality, the synclinic $\mathrm{Sm}-C$ phase is more favorable when $\mu$ is small, whereas the anticlinic $\mathrm{Sm}-C_{A}$ phase is more favorable when $\mu$ is large. Since $g_{1}^{2}$ is also proportional to $\cos ^{-6} \theta$, a phase transition from Sm- $C_{A}$ to $\mathrm{Sm}-C_{A}$ may happen with the increasing tilt angle $\theta$. At the same time, if the dipole moment $\mu$ is very large, a direct transition from $\mathrm{Sm}-C_{A}$ to $\mathrm{Sm}-C_{A}$ may happen.

Terms $\widetilde{F}_{\mathbf{p}}, \Delta \widetilde{F}_{\mathbf{p}}^{(1)}$, and $\Delta \widetilde{F}_{\mathbf{p}}^{(2)}$ in Eq. (35) arise after the minimization of the free energy with respect to polarization 
vectors in every layer. Term $\widetilde{F}_{\mathbf{p}}$ does not include higher than quadratic powers of the small parameter $\sin \theta$ [the first term in Eq. (31)]:

$$
\begin{aligned}
\tilde{F}_{\mathbf{p}}= & -\sin ^{2} \theta \sum_{i=0}^{t-1} \sum_{j=0}^{t-1}\left\{\chi _ { m } \left[c_{p}^{2} f_{j-i}^{(m)}+c_{f}^{2}\left(2 f_{j-i}^{(m)}-\left(f_{j-i-2}^{(m)}+f_{j-i+2}^{(m)}\right)\right.\right.\right. \\
& \left.\times \cos (2 \Delta \varphi))-2 c_{p} c_{f}\left(f_{j-i-1}^{(m)}+f_{j-i+1}^{(m)}\right) \sin \Delta \varphi\right] \cos \varphi_{i j} \\
& +\chi_{m}\left[c_{f}^{2}\left(f_{j-i-2}^{(m)}-f_{j-i+2}^{(m)}\right) \sin (2 \Delta \varphi)-2 c_{p} c_{f}\left(f_{j-i-1}^{(m)}\right.\right. \\
& \left.\left.\left.-f_{j-i+1}^{(m)}\right) \cos \Delta \varphi\right] \sin \varphi_{i j}\right\}-2 c_{f} \sin ^{2} \theta \sum_{i=0}^{t-1}\left\{c _ { f } \operatorname { c o s } \left(\varphi_{i-1, i+1}\right.\right. \\
& \left.+2 \Delta \varphi)+2 c_{p} \sin \left(\varphi_{i, i+1}+\Delta \varphi\right)\right\} .
\end{aligned}
$$

The last two terms in Eq. (38) arose because of the direct coupling between the order parameters within the same smectic layer [the second term in Eq. (22)]. Without them $\widetilde{F}_{\mathbf{p}}$ becomes equal to the corresponding expression obtained in [1] in the absence of the helical rotation $(\Delta \varphi=0)$. At the same time, terms $\Delta \widetilde{F}_{\mathbf{p}}^{(1)}$ and $\Delta \widetilde{F}_{\mathbf{p}}^{(2)}$ in Eq. (35) are completely new. Term $\Delta \widetilde{F}_{\mathbf{p}}^{(1)}$ reflects the anisotropy of the dielectric susceptibility [the second term in Eq. (31)]:

$$
\begin{aligned}
\Delta \tilde{F}_{\mathbf{p}}^{(1)}= & -\sin ^{2} \theta \sum_{i=0}^{t-1} \sum_{j=0}^{t-1}\left\{c_{p}-c_{f}\left[\sin \left(\varphi_{i, i+1}+\Delta \varphi\right)\right.\right. \\
& \left.\left.+\sin \left(\varphi_{i-1, i}+\Delta \varphi\right)\right]\right\}\left\{\operatorname { c o s } \varphi _ { i j } \left[c_{p}\left(\chi_{w} f_{j-i}^{(w)}-\chi_{m} f_{j-i}^{(m)}\right)\right.\right. \\
& \left.-c_{f} \sin \Delta \varphi\left(\chi_{w}\left(f_{j-i-1}^{(w)}+f_{j-i+1}^{(w)}\right)-\chi_{m}\left(f_{j-i-1}^{(m)}+f_{j-i+1}^{(m)}\right)\right)\right] \\
& -c_{f} \sin \varphi_{i j} \cos \Delta \varphi\left(\chi_{w}\left(f_{j-i-1}^{(w)}-f_{j-i+1}^{(w)}\right)\right. \\
& \left.\left.\left.-\chi_{m}\left(f_{j-i-1}^{(m)}-f_{j-i+1}^{(m)}\right)\right)\right]\right\},
\end{aligned}
$$

where $\chi_{w} f_{j-i}^{(w)}-\chi_{m} f_{j-i}^{(m)}$ is proportional to the additional second power of the tilt angle [see Eq. (24)], and thus, $\Delta \widetilde{F}_{\mathbf{p}}^{(1)} \sim \theta^{4}$. Finally, $\Delta \widetilde{F}_{\mathbf{p}}^{(2)}$ is the additional flexoelectric term that contains vertical contribution [the last two terms in Eq. (31)]:

$$
\begin{aligned}
\Delta \widetilde{F}_{\mathbf{p}}^{(2)}= & -c_{f}^{2} \sin ^{4} \theta \sum_{i=0}^{t-1} \sum_{j=0}^{t-1}\left\{2 \chi _ { w } \left[\cos \left(\varphi_{i-1, i}+\Delta \varphi\right)\right.\right. \\
& \left.-\cos \left(\varphi_{i, i+1}+\Delta \varphi\right)\right]\left(f_{j-i-1}^{(w)}-f_{j-i+1}^{(w)}\right) \cos \varphi_{i j} \cos \Delta \varphi \\
& +\chi_{\perp} \cos ^{2} \theta\left(2 f_{j-i}^{(\perp)}-f_{j-i-1}^{(\perp)}-f_{j-i+1}^{(\perp)}\right) \\
& \left.\times \cos \left(\varphi_{i, i+1}+\Delta \varphi\right) \cos \left(\varphi_{j, j+1}+\Delta \varphi\right)\right\} \\
& -2 c_{f}^{2} \sin ^{4} \theta \sum_{i=0}^{t-1}\left\{\cos ^{2}\left(\varphi_{i, i+1}+\Delta \varphi\right)\right. \\
& \left.-\cos \left(\varphi_{i-1, i}+\Delta \varphi\right) \cos \left(\varphi_{i, i+1}+\Delta \varphi\right)\right\}
\end{aligned}
$$

where for simplicity we neglected all the terms proportional to $\sin ^{4} \theta \sin \varphi_{i j}$ which is extremely small in any biaxial subphase, and in any uniaxial phase (including chiral Sm- $C^{*}$ and Sm- $\left.C_{A}^{*}\right)$ the whole term $\Delta \widetilde{F}_{\mathbf{p}}^{(2)}$ is exactly equal to zero, because $\Delta \mathbf{M}_{i}=\mathbf{0}$ [see Eq. (30)].

In paper [1] it was discussed that polarization dependent terms may produce intermediate phases which are different from $\mathrm{Sm}-C$ and $\mathrm{Sm}-C_{A}$. One notes that both chiral dispersion interaction $c_{1}=c_{2}$ and coupling between polar electrostatic and polar dispersion interactions $c_{p} c_{f}$ produce sine functions of the azimuthal angles in the free energy, and therefore may be responsible for the helical rotation.

\section{B. Two types of solutions}

The free energy (35)-(40) should be minimized with respect to all azimuthal angles $\varphi_{i j}$ and $\Delta \varphi$. For simplicity we assume that the tilt angle $\theta$ is the same in every smectic layer and does not depend on $\varphi_{i j}$ and $\Delta \varphi$. Experimental observations confirm this assumption. In contrast to the procedure presented in paper [1], let us examine the two different solutions. One of them would still correspond the biaxial subphases with nonplanar structures which, at the same time, do not deviate strongly from their planar prototypes:

$$
\varphi_{n k}=\sum_{i=n}^{k-1} \alpha_{i}^{0}+\sum_{i=n}^{k-1} \Delta \alpha_{i}
$$

where the angles $\alpha_{i}^{0} \equiv \varphi_{i, i+1}^{0}$ may be equal to 0 or $\pi$ only (i.e., they specify the corresponding planar structure), while the angles $\Delta \alpha_{i} \equiv \Delta \varphi_{i, i+1}$ are assumed to be small (i.e., $\sin \Delta \alpha_{i}$ $\left.\approx \Delta \alpha_{i}, \cos \Delta \alpha_{i} \approx 1\right)$. Additional rotation $\Delta \varphi$ is also assumed to be small (i.e., $\sin \Delta \varphi \approx \Delta \varphi, \cos \Delta \varphi \approx 1$ ). In the case of the biaxial phase, angles $\Delta \alpha_{i}$ and $\Delta \varphi$ describe the deviation from the prototype flat structure. Since minimization of the additional terms $\Delta \widetilde{F}_{\mathbf{p}}^{(1)}$ and $\Delta \widetilde{F}_{\mathbf{p}}^{(2)}$ [see Eqs. (39) and (40)] with respect to these parameters is complicated, and, at the same time, we are examining the linear solution, it is reasonable to ignore these terms (proportional to the additional second power of the small tilt angle in comparison with $\widetilde{F}$ and $\widetilde{F}_{\mathbf{p}}$ ) in the minimization procedure. Finally, minimization of the free energy (35) under the constraints

$$
\varphi_{n k}=\sum_{i=n}^{k-1} \varphi_{i, i+1}, \quad \varphi_{t-1, t}=2 \pi-\sum_{i=0}^{t-2} \varphi_{i, i+1},
$$

which follow directly from periodicity, yields for the biaxial phase:

$$
\sum_{j=0}^{t-2} c_{i j} \Delta \alpha_{j}+c_{i, t-1} \Delta \varphi=q_{i}, \quad i=0, \ldots, t-1,
$$

where

$$
\begin{gathered}
q_{i} \equiv-2 \chi_{m} c_{p} c_{f} \sum_{n=0}^{i} \sum_{k=i+1}^{t-1}\left[f_{k-n-1}^{(m)}-f_{k-n+1}^{(m)}\right] \cos \varphi_{n k}^{0} \\
-\left[\frac{c_{1}}{2} \cos ^{2} \theta-2 c_{p} c_{f}\right]\left(\cos \alpha_{i}^{0}-\cos \alpha_{t-1}^{0}\right), \\
i=0, \ldots, t-2,
\end{gathered}
$$




$$
\begin{aligned}
& q_{t-1} \equiv-2 \chi_{m} c_{p} c_{f} \sum_{n=0}^{t-1} \sum_{k=n+1}^{t-1}\left[f_{k-n-1}^{(m)}+f_{k-n+1}^{(m)}\right] \cos \varphi_{n k}^{0} \\
& -2 \chi_{m} c_{p} c_{f} f_{1}^{(m)} t-\frac{c_{2}}{2} t \sin ^{2} \theta \\
& -\left[\frac{c_{1}}{2} \cos ^{2} \theta-2 c_{p} c_{f}\right]_{n=0}^{t-1} \cos \alpha_{n}^{0} \\
& c_{i j} \equiv-b \sin ^{2} \theta-\frac{a}{2} \cos ^{2} \theta \cos \alpha_{t-1}^{0} \\
& +\chi_{m} \sum_{n=0}^{\min (i, j)} \sum_{k=\max (i, j)+1}^{t-1}\left\{c_{p}^{2} f_{k-n}^{(m)}+c_{f}^{2}\left[2 f_{k-n}^{(m)}-\left(f_{k-n-2}^{(m)}\right.\right.\right. \\
& \left.\left.\left.+f_{k-n+2}^{(m)}\right)\right]\right\} \cos \varphi_{n k}^{0}+\left(c_{f}^{2}-\frac{a_{1}}{2}\right)\left(\delta_{i 0} \delta_{j 0} \cos \varphi_{-1,1}^{0}\right. \\
& \left.+\delta_{i, t-2} \delta_{j, t-2} \cos \varphi_{t-2, t}^{0}\right)+\Delta c_{i j}, \quad i, j=0, \ldots, t-2, \\
& \Delta c_{i j} \equiv 0, \quad j \neq i, \quad j \neq i \pm 1, \\
& \Delta c_{i i} \equiv-b \sin ^{2} \theta-\frac{a}{2} \cos ^{2} \theta \cos \alpha_{i}^{0}+\left(c_{f}^{2}-\frac{a_{1}}{2}\right) \\
& \times\left(\delta_{i 0} \cos \varphi_{i-1, i+1}^{0}+\delta_{i, t-2} \cos \varphi_{i, i+2}^{0}\right), \\
& \Delta c_{i, i \pm 1}=\Delta c_{i \pm 1, i} \equiv\left(c_{f}^{2}-\frac{a_{1}}{2}\right) \cos \left(\alpha_{i}^{0}+\alpha_{i \pm 1}^{0}\right), \\
& c_{i, t-1}=c_{t-1, i} \equiv-\frac{a}{2} \cos ^{2} \theta\left(\cos \alpha_{i}^{0}-\cos \alpha_{t-1}^{0}\right) \\
& -2 \chi_{m} c_{f}^{2} \sum_{n=0}^{i} \sum_{k=i+1}^{t-1}\left[f_{k-n-2}^{(m)}-f_{k-n+2}^{(m)}\right] \cos \varphi_{n k}^{0},+\left(2 c_{f}^{2}-a_{1}\right) \\
& \times\left(\delta_{i 0} \cos \varphi_{i-1, i+1}^{0}+\delta_{i, t-2} \cos \varphi_{i, i+2}^{0}\right), \\
& i=0, \ldots, t-2, \\
& c_{t-1, t-1} \equiv-b t \sin ^{2} \theta-\frac{a}{2} \cos ^{2} \theta \sum_{n=0}^{t-1} \cos \alpha_{n}^{0} \\
& -4 \chi_{m} c_{f}^{2} \sum_{n=0}^{t-1} \sum_{k=n+1}^{t-1}\left[f_{k-n-2}^{(m)}+f_{k-n+2}^{(m)}\right] \cos \varphi_{n k}^{0} \\
& -4 \chi_{m} c_{f}^{2} f_{2}^{(m)} t+2\left(2 c_{f}^{2}-a_{1}\right) \sum_{n=0}^{t-1} \cos \varphi_{i-1, i+1}^{0} \text {. }
\end{aligned}
$$
easy to study $\mathrm{Sm}-C_{\alpha}^{*}$ not as a solution of type (41), but as an additional solution:

$$
\varphi_{n k}=2 \pi \eta \frac{k-n}{t}, \quad \Delta \varphi=0
$$

where $\eta$ is some value between $-t / 2$ and $t / 2$. Substituting Eq. (46) into Eq. (35) one finds $\eta$ corresponding to the minimum free energy for any particular period $t$. Comparing this free energy with the one corresponding to the solution of Eqs. (43) one finds whether the uniaxial or the biaxial phase is more stable.

Finally, the free energies of phases with different periods $t$ are compared with each other to select the one that corresponds to a global minimum for a particular choice of the model parameters. We have performed the corresponding numerical calculations for several integer numbers $t$ and $\eta$. The results will be presented in Sec. V.

Concluding this section we should note that without neglecting the biaxial ordering $\sigma$ in Eq. (29) we could not obtain the linear set of equations for polarizations (23). At the same time, all the terms taken into account in the free energy (35) essentially depend on the molecular tilt and disappear without tilt. On the contrary, the neglected terms (which are proportional to the small biaxial ordering parameter $\sigma$ ) remain nonzero in the absence of tilt and, as it will be shown in Sec. IV, may produce different regime of rotation (classified as $\mathrm{Sm}-C_{\alpha}^{*}$ ) in the case of a very small tilt.

\section{IV. "FINE TUNING" OF THE FREE ENERGY NEAR THE TRANSITION BETWEEN SMECTICS C AND A: ALPHA AND DE VRIES' PHASES}

Now let us investigate the behavior of the liquid crystal in the vicinity of the $\mathrm{Sm}-C^{*}-\mathrm{Sm}-A^{*}$ transition point. It is difficult to take into account the biaxial ordering $\sigma$ in an arbitrary intermediate phase because an additional coupling of the four different projections of polarizations on the coordinate axes will arise in the free energy, and thus, equations for polarizations will become nonlinear. At the same time, the biaxial ordering $\sigma$ is expected to be essential only in the case of the small tilt angles, where the arising phase is known to be uniaxial and the polarization vector is known to be perpendicular to the tilt plane in every layer. In this particular case Eqs. (23) for polarizations do not change, and one obtains only the following changes in the coefficients $b$ and $c_{2}$ [see Eqs. (37)]:

$$
\begin{gathered}
b \rightarrow \tilde{b}=b\left(1-\sigma^{2}\right)+\Delta b \sigma^{2} \cos ^{2} \theta / \sin ^{4} \theta, \\
c_{2} \rightarrow \widetilde{c}_{2}=c_{2}\left(1-\sigma^{2}\right)+\Delta c_{2} \sigma^{2} \cos ^{2} \theta / \sin ^{4} \theta,
\end{gathered}
$$




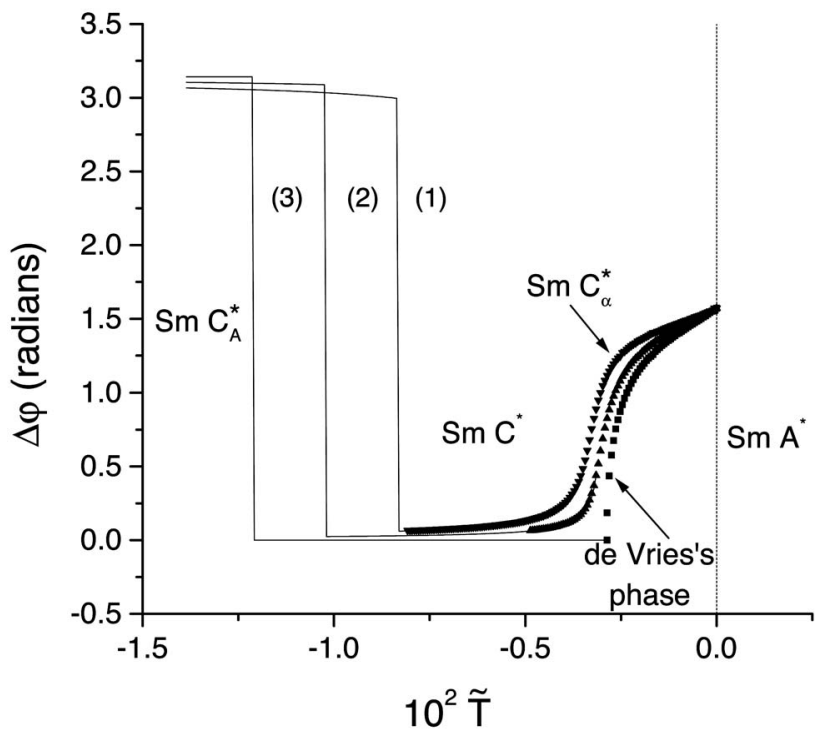

FIG. 3. Helical rotation angle in $\mathrm{Sm}-C_{A}^{*}, \mathrm{Sm}-C^{*}$, and $\mathrm{Sm}-C_{\alpha}^{*}$ as a function of temperature in the case $B_{0} / B_{1}=-0.1, \alpha / B_{1}=-0.5, \sigma$ $=0.2, \ell / d=4, \widetilde{v}_{1}=-0.622, \widetilde{v}_{3}=1.72, \Delta \widetilde{v}_{1}=0, \Delta \widetilde{v}_{5}=0, \mu_{\mathrm{ef}}=0.77$, and the following set of parameters $\widetilde{v}_{5}, \widetilde{v}_{6}$, and $Q_{\text {ef }}$ (see definitions of all parameters in Sec. V): (1) $\widetilde{v}_{5}=-1.5 \times 10^{-2}, \widetilde{v}_{6}=-0.15, Q_{\text {ef }}=1.04$; (2) $\widetilde{v}_{5}=-7.5 \times 10^{-3}, \widetilde{v}_{6}=-0.11, Q_{\mathrm{ef}}=0.736$; and (3) $\widetilde{v}_{5}=\widetilde{v}_{6}=Q_{\mathrm{ef}}=0$. In the last case direction of the rotation is undetermined, and $\mathrm{Sm}-C_{\alpha}^{*}$ becomes the de Vries' phase.

where $\Delta b=\Delta \widetilde{v}_{1}+4 g_{1}^{2}$ generally consists of the dispersion (defined here as $\Delta \widetilde{v}_{1}$ ) and electrostatic (equal to $4 g_{1}^{2}$ ) contributions, and $\Delta c_{2}=\Delta \widetilde{v}_{5}$ consists only of the dispersion contribution (defined here as $\Delta \widetilde{v}_{5}$ ). It was discussed in Sec. III that coefficient $b$ is negative that causes the synclinic or anticlinic state in the absence of chirality- and polarization-dependent terms. At the same time, one notes that the electrostatic part of $\Delta b$ is positive, and thus, in the case of the small angles $\theta$ the total coefficient $\tilde{b}$ may become positive if $\sigma>0$, and the phase with the helical rotation angle $\Delta \varphi$ essentially different from 0 and $\pi$ may arise even in the case of nonchiral nonpolar molecules $\left(c_{1}=\widetilde{c}_{2}=c_{p}=c_{f}=0\right)$. In Fig. 3 the helical rotation angle is presented as a function of the temperature for different values of the conventional chirality (determined by parameter $\left.\widetilde{v}_{5} \equiv c_{1}=\widetilde{c}_{2}\right)$ and of the combined chirality [determined by a combination of the flexoelectric $\left(c_{f} \sim Q_{\mathrm{ef}}\right)$ and piezoelectric $\left(c_{p} \sim \widetilde{v}_{6} \equiv v_{6} /\left(k_{B} T^{*}\right)\right)$ effects]. Taking into account that the helical rotation may, in principle, be large, and, consequently, the perturbation scheme (43) may work badly, we also made a direct calculation of the periodicity according to the scheme (46) using integer numbers up to 100 for $t$ and integer numbers between $-t / 2$ and $t / 2$ for $\eta$. Perturbation scheme (43) (see solid lines in Fig. 3) works better in the case of the small helical rotation angles, whereas direct scheme (46) (see triangles and squares) works better for the large helical rotation angles. But one notes that both schemes give approximately the same helical rotation angles at a large scale. We note the fact that a direct calculation of the periodicity is quite a long computer procedure, and the perturbation scheme appears to be very helpful and accurate enough. If $\widetilde{v}_{5} \neq 0$ and $c_{p} c_{f} \neq 0$ [see curves (1) and (2) in Fig. 3], the helical rotation angle is slightly different from 0 in $\mathrm{Sm}-C^{*}$ and from $\pi$ in Sm- $C_{A}^{*}$. One notes, however, that in the vicinity of the transition into $\mathrm{Sm}-A^{*}\left[\widetilde{T} \equiv\left(T-T^{*}\right) / T^{*} \leq 0\right]$ the helical rotation angle rapidly increases with the temperature increasing, and finally becomes equal to $\pi / 2$ in Sm- $A^{*}$. This phase with large helical rotation angle arising near the transition point may be interpreted as $\mathrm{Sm}-C_{\alpha}^{*}$ (by contrast to conventional $\mathrm{Sm}-C^{*}$ that is observed for lower temperatures). The same effect was achieved in the phenomenological model [15] where a significantly large term in the free energy proportional to $\cos ^{2} \Delta \varphi$ was introduced artificially. In our model the analogous term (proportional to the coefficient b) remains nonzero in the vicinity of $\theta=0$ because of the biaxial nonpolar ordering. In contrast to the other terms in the free energy, it is not proportional to various positive powers of $\sin \theta$, and thus, must not be large initially to produce $\mathrm{Sm}-C_{\alpha}^{*}$. The direction of the rotation (in other words, the sign of the helical rotation angle) is determined by the signs of the chiral terms $\widetilde{v}_{5}$ and $c_{p} c_{f}$. At the same time, for the case without any chiral terms [see curve (3) in Fig. 3] the nonzero helical rotation angle is still favorable if the tilt is very small. However, the direction of this rotation is undetermined. In other words, a system prefers to rotate just in any direction more than remain without rotation. This situation may be interpreted as the de Vries's phase [10] where the rotation does not have regular direction. The appearance of this phase seems to have no easy explanation at first glance. At the same time, we can explain the origin of this phase coming back to the initial expressions for the free energy (1), (5), and (6) One notes that positive $\Delta b$ (which is responsible for the de Vries's phase) arises from the last term in Eq. (6), which is positive since $U_{M F}^{(i)}$ is negative. Indeed, a new polarization dependent term [see the first term in Eq. (29)] arise only if the distribution function $f_{i}(\psi)$ under the integral is different from 1, and thus the remaining terms in Eq. (6) do not participate in $\Delta b$, although they dominate $b$. Now the only question arises as why the last term in Eq. (6) [or in Eq. (5)] is positive? This term consists both of the energy and the entropy contributions, but its positive sign is determined only by the entropy contribution that dominates in this term, whereas the energy participates mostly in the first term of Eq. (5) which does not contribute into $\Delta b$ Thus, at least in our theory, the de Vries's phase is a pure product of the entropy effects in the presence of the biaxial nonpolar ordering, that seems to be consistent with the "chaotic" character of this phase. It arises when the energy contribution is extremely small due to a small tilt angle.

The Sm- $C_{\alpha}^{*}$ phase has exactly the same origin and actually differs from the de Vries's phase only by the small chiral terms in the free energy that select between the two directions of the rotation, and thus produce the regular rotation from layer to layer. The direction of the rotation in the de Vries's phase may be irregular, or may be determined by rather small effects that are not considered here. The behavior of the rotation in $\mathrm{Sm}-C_{\alpha}^{*}$ may be different depending on the chirality and polarization constants. Although the real signs of the constants $c_{1}=c_{2}, \Delta c_{2}$, and $c_{p} c_{f}$ may be determined only from the more accurate molecular model, it is quite natural to suppose that if molecules are regularly 


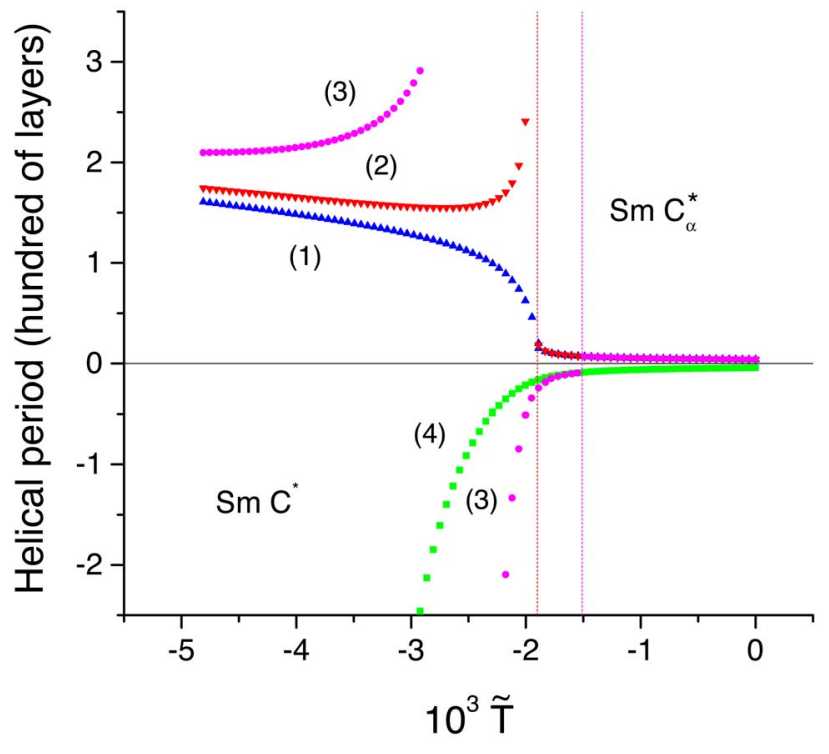

FIG. 4. (Color online) Helical period in Sm- $C^{*}$ and $\mathrm{Sm}-C_{\alpha}^{*}$ as a function of temperature in the case $B_{0} / B_{1}=-0.1, \alpha / B_{1}=-0.5, \sigma$ $=0.2, \quad \ell / d=4, \quad \widetilde{v}_{1}=-0.622, \quad \Delta \widetilde{v}_{1}=0, \quad \widetilde{v}_{3}=1.62, \quad \widetilde{v}_{5}=-1.5 \times 10^{-2}$, $\widetilde{v}_{6}=-0.15, \mu_{\mathrm{ef}}=0.77, Q_{\mathrm{ef}}=1.04$ and different values of parameter $\Delta \widetilde{v}_{5}$ (see definitions of all parameters in Sec. V): (1) $\Delta \tilde{v}_{5}=10^{-2}$; (2) $\Delta \widetilde{v}_{5}=1.25 \times 10^{-2}$; (3) $\Delta \widetilde{v}_{5}=1.38 \times 10^{-2}$; and (4) $\Delta \widetilde{v}_{5}=2 \times 10^{-2}$.

twisted, then both coefficients of the chiral dispersion interaction $c_{1}=c_{2}$ and of the combined chiral interaction $c_{p} c_{f}$ induced by polarization have the same sign. At the same time, the sign of the addition $\Delta c_{2}$ appears to be opposite if one takes all chiral spherical invariants with the same sign, because the terms $P_{i}^{(\alpha)} P_{i}^{(\beta)}$ and $n_{i}^{(\alpha)} n_{i}^{(\beta)}$ in Eq. (29) have opposite signs. Thus let us suppose $c_{1}=c_{2}=\widetilde{v}_{5}<0, c_{p} c_{f} \sim \widetilde{v}_{6} Q_{\text {ef }}$ $<0$, and $\Delta c_{2}=\Delta \tilde{v}_{5}>0$. Then if the absolute value of $\Delta \tilde{v}_{5}$ is small with respect to that of the remaining chirality coefficients (both conventional and combined), a continuous (although very sharp) transition from $\mathrm{Sm}-C^{*}$ to $\mathrm{Sm}-C_{\alpha}^{*}$ happens [see curve (1) in Fig. 4]. With the increasing $\left|\Delta \widetilde{v}_{5} / \widetilde{v}_{5}\right|$ the helical period may begin to diverge in the $\mathrm{Sm}-C^{*}-\mathrm{Sm}-C_{\alpha}^{*}$ transition point [see curve (2) in Fig. 4] or (for larger $\left.\left|\Delta \widetilde{v}_{5} / \widetilde{v}_{5}\right|\right)$ before this transition point [i.e., within $\mathrm{Sm}-C^{*}$, see curve (3) in Fig. 4]. For essentially large $\left|\Delta \widetilde{v}_{5} / \widetilde{v}_{5}\right|$ [see curve (4) in Fig. 4] the direction of the rotation may remain negative in both $\mathrm{Sm}-C^{*}$ and $\mathrm{Sm}-C_{\alpha}^{*}$. Thus, in the cases presented in curves (1) and (4) a continuous transition from Sm- $C^{*}$ to $\mathrm{Sm}-C_{\alpha}^{*}$ must be observed. The divergence of the helical period within the Sm- $C^{*}-\mathrm{Sm}-C_{\alpha}^{*}$ range is observed experimentally, for example, in [16], whereas the continuous modification is observed, for example, in $[17,18]$. Finally one notes that there may not exist $\mathrm{Sm}-C_{\alpha}^{*}$ or its temperature range may be narrow or hardly noticeable, if the biaxial ordering $\sigma$ is equal to zero or very small. Generally, the behavior of the helical period in $\mathrm{Sm}-\mathrm{C}^{*}$ is very similar to that presented in [19] and is described well by perturbation scheme (43). In the case of Sm- $C^{*}$ it yields the following simple expression for the helical rotation angle:

$$
\Delta \varphi^{1}=\frac{2\left(\frac{\chi_{m}}{1+2 g_{m}}-1\right) c_{p} c_{f}+\frac{c_{1}}{2} \cos ^{2} \theta+\frac{\widetilde{c}_{2}}{2} \sin ^{2} \theta}{\tilde{b} \sin ^{2} \theta+\left(\frac{a}{2}+2 a_{1}\right) \cos ^{2} \theta+4\left(\frac{\chi_{m}}{1+2 g_{m}}-1\right) c_{f}^{2}},
$$

where index " 1 " denotes $q T=1$. The expression for the helical rotation angle is also very simple in $\mathrm{Sm}-C_{A}^{*}(q T=0)$ :

$$
\Delta \varphi^{0}=-\frac{2\left(\frac{\chi_{m}}{1-2 g_{m}}-1\right) c_{p} c_{f}+\frac{c_{1}}{2} \cos ^{2} \theta-\frac{\widetilde{c}_{2}}{2} \sin ^{2} \theta}{\tilde{b} \sin ^{2} \theta-\left(\frac{a}{2}-2 a_{1}\right) \cos ^{2} \theta+4\left(\frac{\chi_{m}}{1-2 g_{m}}-1\right) c_{f}^{2}} .
$$

But Sm- $C_{\alpha}^{*}$ does not obey the scheme (43) and Eq. (48). If $\sigma>0$ the helical rotation remains even in Sm- $A^{*}$. The question arises, what rotates in this phase? Indeed, polarization disappears in the untilted phase, since it is proportional to $\sin \theta$. Nevertheless, the biaxial nonpolar ordering still remains, and the director of this ordering is parallel to polarization that arose previously in the tilted uniaxial phase. Parameter $\sigma$ may, in principle, be obtained by minimization of the extended free energy that also contains the higher powers of polarization vectors. This task, however, seems to be very difficult, and what is more important, takes even more parameters that are undetermined. At the same time, it is clear that parameter $\sigma$ does not change very much within the investigated temperature interval, since the terms containing it are almost independent of the molecular tilt. Therefore we simply considered $\sigma$ as an additional parameter in our theory.

\section{PHASE DIAGRAMS AND DISCUSSION}

It is well-known that the temperature range covering all the intermediate smectic phases is usually less than $10 \mathrm{~K}$. At the same time, the tilt angle $\theta$ is known to change dramatically within this temperature range. In contrast to paper [1], let us assume at zeroth approximation that the only parameter depending on the temperature is the tilt angle. In this case the phase diagrams may be plotted in terms of the variable tilt angle $\theta$ even without introducing the temperature. At the same time, it is not difficult to introduce a model expression for the temperature variation of the tilt angle. Let us assume in addition that the tilt angle is determined mostly by the interaction of molecules located within the same smectic layers and expand the corresponding free energy $F_{0}(\theta)$ in Taylor series first with respect to $\sin \theta$ at point $\sin \theta=0$ and then with respect to the dimensionless declination $\widetilde{T} \equiv(T$ $\left.-T^{*}\right) / T^{*}$ from the $\mathrm{Sm}-A^{*}-\mathrm{Sm}-C^{*}$ transition temperature $T^{*}$ at point $\widetilde{T}=0$ :

$$
F_{0}(\theta)=\alpha \widetilde{T} \sin ^{2} \theta+\left(B_{0}+B_{1} \widetilde{T}\right) \sin ^{4} \theta,
$$

where the coefficients $\alpha, B_{0}$, and $B_{1}$ depend on the molecular model. The behavior of the tilt angle is quite standard though: it arises at some temperature $T^{*}$, then increases rapidly with temperature decreasing, and finally saturates some 
degrees below $T^{*}$. Minimizing Eq. (50) with respect to $\sin \theta$ one obtains:

$$
\sin ^{2} \theta=-\frac{\alpha \widetilde{T}}{2\left(B_{0}+B_{1} \widetilde{T}\right)} .
$$

The desired behavior is easily achieved when $\alpha>0, B_{0}>0$, and $B_{1}<0$. Indeed, the ratio $-B_{1} / B_{0}$ regulates the "speed" of saturation. Saturation of the tilt angle is very fast if the ratio $-B_{1} / B_{0}$ is large. The ratio $-\alpha /\left(2 B_{1}\right)$ corresponds to the square of the sine function of the saturated tilt angle.

Now let us define the remaining independent parameters in the case of the strong positional correlation of molecules in the same and in the neighboring layers $\left(\rho\left\langle g_{i, j}\right\rangle \approx 1\right)$. The most important parameters are the dimensionless dipole and quadrupole moments:

$$
\mu_{\mathrm{ef}} \equiv \mu \sqrt{\frac{1}{k_{B} T^{*} d^{3}}}, \quad Q_{\mathrm{ef}} \equiv Q \sqrt{\frac{1}{k_{B} T^{*} d^{5}}},
$$

that participate in $g_{0}=\mu_{\mathrm{ef}}^{2} / 8, g_{1}=\left[d /\left(h_{1} \cos \theta\right)\right]^{3} \mu_{\mathrm{ef}}^{2} / 4$ and $c_{f}=-3\left(d / h_{2}\right)^{4} Q_{\mathrm{ef}} \mu_{\mathrm{ef}} / 2$ [see Eqs. (26) and (10)]. In addition one needs the dispersion coefficients $\widetilde{v}_{i} \equiv v_{i} /\left(k_{B} T^{*}\right)(i=1,3$, 5, and 6) and $\Delta \widetilde{v}_{i} \equiv \Delta v_{i} /\left(k_{B} T^{*}\right)(i=1$ and 5) that participate in $a, b, c_{1}=c_{2}$ [see Eqs. (37) and (47)] and in $c_{p}$ [see Eq. (19)].

In papers $[6,13]$ it was discussed that in the absence of polarization and chirality effects the molecular transverse terminal dipole moment $\mu_{\mathrm{ef}}$ favors the anticlinic state, whereas a combination of the dispersion coefficients $3 \widetilde{v}_{1}+\widetilde{v}_{3}$ favors the synclinic state. In paper [1] it was noticed that the interplay between various intermediate smectic phases is managed by the flexoelectric effect (proportional to molecular quadrupole moment $Q_{\mathrm{ef}}$ ) and the piezoelectric effect (proportional here to polar dispersion coefficient $\widetilde{v}_{6}$ ). Let us therefore introduce the reduced dipole moment $\mu_{\mathrm{ef}}^{4} /\left|4\left(3 \widetilde{v}_{1}+\widetilde{v}_{3}\right)\right|$ and the reduced quadrupole moment $\left|Q_{\mathrm{ef}} /\left(14 \widetilde{v}_{6}\right)\right|$, and consider the reduced dipole-temperature and the reduced quadrupole-temperature phase diagrams. Taking into account that in numerous smectic materials the transverse dipole moment is associated with the same polar atomic group (for example, $\mathrm{CH}_{3}$ ), let us fix parameter $\mu_{\mathrm{ef}}$ in our reduced dipole-temperature phase diagram and consider the dispersion contribution $3 \widetilde{v}_{1}+\widetilde{v}_{3}$ as the variable. The corresponding phase diagram in the case of molecules with small reduced quadrupole moment is presented in Fig. 5(a), where the uniaxial phases fill all the "space" of the diagram. In this figure the uniaxial phases are marked by numbers of layers per one turn $p_{s l} \equiv t / \eta$, and the red thick line detaches $\mathrm{Sm}-C_{A}^{*}, \mathrm{Sm}-C^{*}$, and $\mathrm{Sm}-C_{\alpha}^{*}$ (let us call this line the phase transition border). In the presence of the nonpolar biaxial ordering (see Sec. IV) the phase transition border becomes cap-shaped. Below and above the phase transition border the periodicity of uniaxial phase modifies continuously (only periodicities equal to the ratios of some integer numbers are shown in Fig. 5). It is equal to several smectic layers in $\mathrm{Sm}-C_{\alpha}^{*}$, to several hundreds of layers in $\mathrm{Sm}-C^{*}$, and to approximately two layers in $\mathrm{Sm}-C_{A}^{*}$. For different values of parameter $\mu_{\mathrm{ef}}^{4}|| 3 \widetilde{v}_{1}+\widetilde{v}_{3} \mid$ the value of parameter $p_{s l}$ in $\mathrm{Sm}-C_{\alpha}^{*}$ may decrease or increase with the increasing tem-
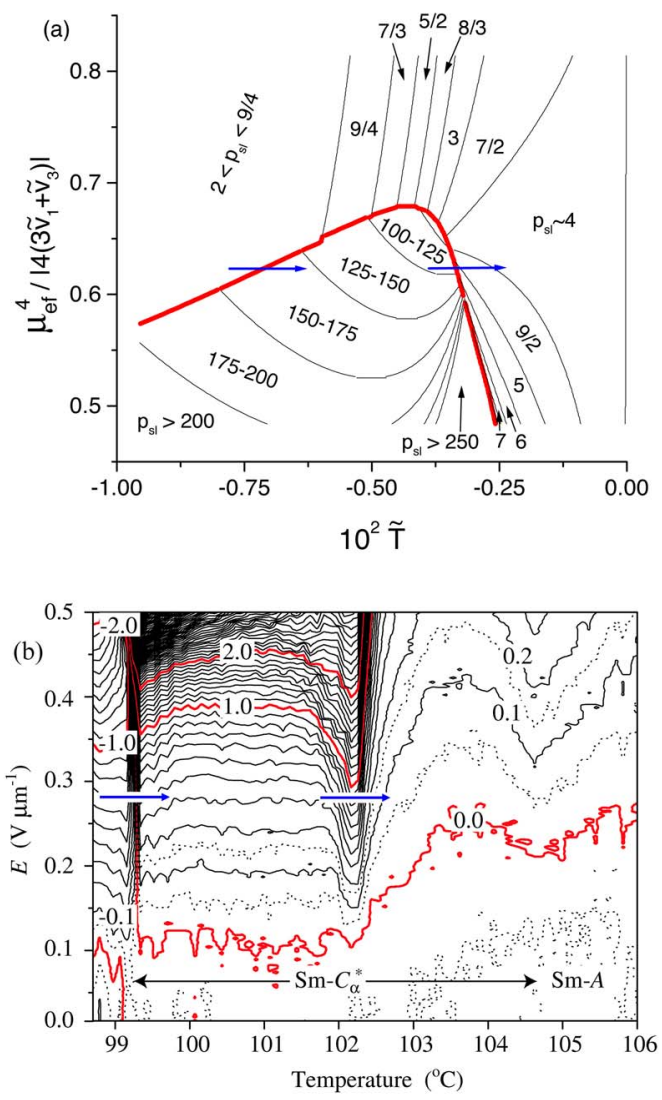

FIG. 5. (Color online) (a) Theoretical reduced dipoletemperature phase diagram in the case $B_{0} / B_{1}=-0.1, \alpha / B_{1}=-0.5$, $\sigma=0.2, \ell / d=4, \widetilde{v}_{1}=-0.622, \Delta \widetilde{v}_{1}=0, \quad \widetilde{v}_{5}=-1.5 \times 10^{-2}, \Delta \widetilde{v}_{5}=1.75$ $\times 10^{-2}, \widetilde{v}_{6}=-0.15, \mu_{\mathrm{ef}}=0.77$, and $Q_{\mathrm{ef}}=1.04$; and (b) Experimental electric-field-induced birefringence diagram for MHPOCBC reproduced from [6]. Blue arrows in both figures seem to correspond to the same transitions.

perature. There is an interesting correlation between the value of parameter $p_{s l}$ in $\mathrm{Sm}-C_{\alpha}^{*}$ and its derivative with respect to the temperature: it increases with the temperature increasing if it is small $\left(p_{s l} \lesssim 4\right)$, and decreases if it is large $\left(p_{s l} \geq 4\right)$. This correlation is in complete correspondence with the experiment $[20,21]$. The blue arrows in Fig. 5(a) show the first order phase transitions between $\mathrm{Sm}-C_{A}^{*}$ and $\mathrm{Sm}-C^{*}$, and between $\mathrm{Sm}-C^{*}$, and $\mathrm{Sm}-C_{\alpha}^{*}$, although a continuous modification from $\mathrm{Sm}-C^{*}$ to $\mathrm{Sm}-C_{\alpha}^{*}$ is also possible (see Sect. IV), which is similar to the liquid-gas coexistence above critical point. One notes from Fig. 5(a) that near the peak of the phase transition border $\mathrm{Sm}-\mathrm{C}^{*}$ may be very narrow and its periodicity may be quite small, so that sometimes $\mathrm{Sm}-C^{*}$, and $\mathrm{Sm}-C_{\alpha}^{*}$, may be interpreted as "two kinds of Sm- $C_{\alpha}^{*}$," (see [6], where, however, these two kinds were associated with crossing the left "arm" of the phase transition border, because we could not yet predict the existence of the right "arm"). The experimental electric-field-induced birefringence diagram for MHPOCBC reproduced from [6] is presented in Fig. 5(b), where the blue arrows (now correctly) represent the transitions between $\mathrm{Sm}-C_{A}^{*}, \mathrm{Sm}-C^{*}$, and $\mathrm{Sm}-C_{\alpha}^{*}$.

When the molecular quadrupole moment $\left|Q_{\text {ef }}\right|$ is large the left "arm" of the phase transition border breaks into two 

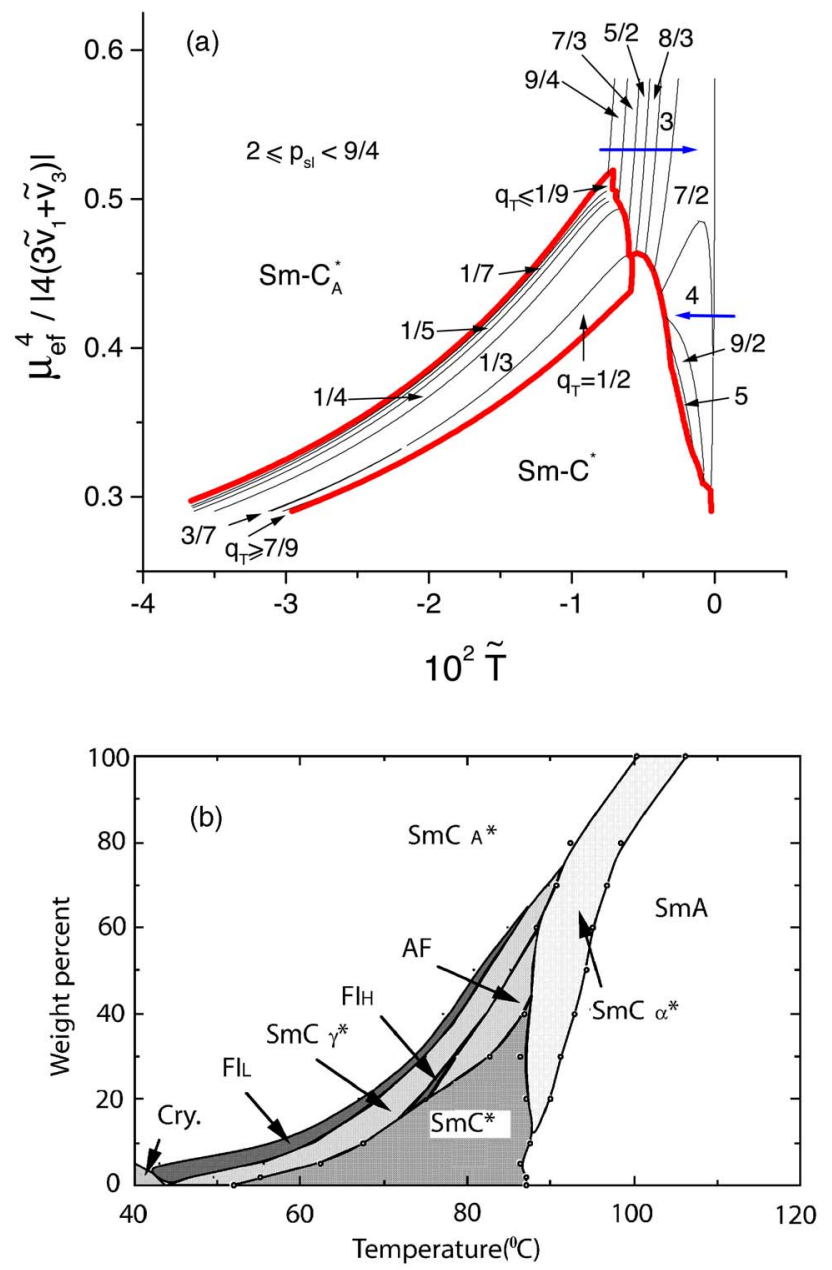

FIG. 6. (Color online) (a) Theoretical reduced dipoletemperature phase diagram in the case $B_{0} / B_{1}=-0.1, \alpha / B_{1}=-0.5$, $\sigma=0.2, \quad \ell / d=4, \quad \widetilde{v}_{1}=-0.622, \quad \Delta \widetilde{v}_{1}=0, \quad \widetilde{v}_{5}=-1.5 \times 10^{-2}, \Delta \widetilde{v}_{5}=2.5$ $\times 10^{-2}, \widetilde{v}_{6}=-4.74 \times 10^{-2}, \mu_{\mathrm{ef}}=0.77$, and $Q_{\mathrm{ef}}=3.29$; and (b) Experimental phase diagram reproduced from [9]. Molecules of two compounds MHPOCBC and MHPOOCBC seem to have different values of parameter $3 \widetilde{v}_{1}+\widetilde{v}_{3}$.

lines, and a sequence of biaxial subphases arises in the area between these two lines [see Fig. 6(a), where the red thick lines detach the biaxial phases from the uniaxial ones and $\mathrm{Sm}-C^{*}$ from $\mathrm{Sm}-C_{\alpha}^{*}$, the blue arrows show the increasing with temperature pitch in $\mathrm{Sm}-C_{\alpha}^{*}$ if $p_{s l} \lesssim 4$ and the decreasing pitch if $p_{s l} \gtrsim 4$, the uniaxial phases are still marked by $p_{s l}$, and the biaxial phases are marked by $q_{T}$ denoting the number of synclinic pairs per one period $t]$. The well-known $\mathrm{Sm}-C_{A}^{*}(1 / 3)[\mathrm{FI} 1]$ and $\mathrm{Sm}-C_{A}^{*}(1 / 2)[\mathrm{FI} 2]$ appear to be the broadest phases in the temperature range, though different biaxial subphases with $q_{T}<1 / 3$ and even with $1 / 3<q_{T}$ $<1 / 2$ and $q_{T}>1 / 2$ [22] arise within the small temperature ranges. One notes that in the case of large $\mu_{\mathrm{ef}}^{4} /\left|3 \tilde{v}_{1}+\widetilde{v}_{3}\right|$ (let us call it case 1$) \mathrm{Sm}-C_{A}^{*}(1 / 3)$ and $\mathrm{Sm}-C_{A}^{*}(1 / 2)$ arise in approximately equal temperature ranges, and simultaneously the higher temperature $\mathrm{Sm}-C^{*}$ arises in relatively small temperature range, and in addition a continuous modification from $\mathrm{Sm}-C^{*}$ to $\mathrm{Sm}-C_{\alpha}^{*}$ similar to that presented in curve (1) in Fig. 4 (that may be interpreted as the absence of Sm- $C^{*}$ ) is rather possible in this case because of the relatively small periodicity in $\mathrm{Sm}-C^{*}$. This situation tentatively corresponds to experimental data for $10 \mathrm{OTBBB} 1 \mathrm{M} 7$ presented in [12] and for MHPBC presented in [11]. At the same time, in the case of small $\mu_{\mathrm{ef}}^{4} f / 3 \widetilde{v}_{1}+\widetilde{v}_{3} \mid$ (case 2) $\mathrm{Sm}-C_{A}^{*}(1 / 2)$ is essentially narrower than $\mathrm{Sm}-C_{A}^{*}(1 / 3)$ (and sometimes is absent), $\mathrm{Sm}-C^{*}$ is very broad, and the helical pitch in $\mathrm{Sm}-C^{*}$ is essentially long. This corresponds to experimental data for MHPOBC and MHDDOPTCOB presented in the same papers. In our theory these three output parameters [relative range of $\mathrm{Sm}-C_{A}^{*}(1 / 2)$, relative range of $\mathrm{Sm}-C^{*}$ (both with respect to $\left.\mathrm{Sm}-C_{A}^{*}(1 / 3)\right)$, and the value of helical pitch in $\left.\mathrm{Sm}-C^{*}\right]$ appear to depend on the value $\mu_{\mathrm{ef}}^{4} /\left|3 \widetilde{v}_{1}+\widetilde{v}_{3}\right|$ which is an input molecular parameter. Unfortunately it is difficult to estimate this value experimentally. Nevertheless, the simultaneous change of our output parameters with the change of value $\mu_{\mathrm{ef}}^{4} /\left|3 \widetilde{v}_{1}+\widetilde{v}_{3}\right|$ exactly corresponds to the simultaneous change of the same experimental parameters with the choice of different materials. We believe that this simultaneous change, as well as correlation between $p_{s l}$ in $\mathrm{Sm}-C_{\alpha}^{*}$ and its derivative with respect to the temperature discussed above, is not just a coincidence, but a regular confirmation of our theory. Moreover, the phase diagram presented in Fig. 6(a) as a whole resembles the experimental result presented in Fig. 6(b), where molecules of two compounds MHPOCBC and MHPOOCBC have the same terminal transverse dipole moment associated with polar group $\mathrm{CH}_{3}$, but seem to have different values of the dispersion parameter $3 \widetilde{v}_{1}+\widetilde{v}_{3}$ favoring the synclinic state.

Let us outline the theoretical mechanism of the change of the mentioned output parameters. The $\mathrm{Sm}-C^{*}$ phase extends over a smaller temperature interval in case 1 and over a larger temperature interval in case 2 , because the phase transition border is regulated by the value $g_{1}^{2} /\left|3 \tilde{v}_{1}+\tilde{v}_{3}\right|$ $=\mu_{\mathrm{ef}}^{4} /\left(\left|3 \tilde{v}_{1}+\tilde{v}_{3}\right| \cos ^{6} \theta\right)$ [see Eqs. (36) and (37)], and thus, its left "arm" moves to the smaller tilt angles $\theta$ (in other words, to the higher temperatures) with the increasing $\mu_{\mathrm{ef}}^{4} / \mid 3 \widetilde{v}_{1}$ $+\widetilde{v}_{3} \mid$. The helical pitch is short in case 1 and long in case 2 , because the flexoelectric constant $c_{f}$ is proportional to $\mu_{\mathrm{ef}}$. Finally, let us understand why $\mathrm{Sm}-C_{A}^{*}(1 / 2)$ is broad in case 1 and narrow in case 2 . One notes that the additional flexoelectric term $-\mathbf{n}_{i}\left(\mathbf{n}_{i} \cdot \Delta \mathbf{n}_{i \pm 1}\right)$ in the free energy [see discussion after Eq. (18)] produces the last term $\Delta \widetilde{F}_{\mathbf{p}}^{(2)}$ in the free energy (35). Among subterms with different $i$ and $j$ participating in this term [see Eq. (40)], let us collect the ones proportional to the coefficients $f_{1}^{(w)}$ and $f_{1}^{(\perp)}$ (and thus, giving the largest contribution to the free energy). One easily obtains the function proportional to $-\cos \varphi_{i-1, i} \cos \varphi_{i, i+1}$ which does not distinguish $\mathrm{Sm}-C^{*}$ from $\mathrm{Sm}-C_{A}^{*}$, but at the same time it does not favor $\mathrm{Sm}-C_{A}^{*}(1 / 2)$, which is neither synclinic-nor anticlinic-like. This subphase appears to be suppressed by the neighboring phases $\left[\mathrm{Sm}-C_{A}^{*}(1 / 3)\right.$ and $\left.\mathrm{Sm}-C^{*}\right]$. Before consideration of the vertical (perpendicular to the smectic layer plane) flexoelectric polarization $\mathrm{Sm}-C_{A}^{*}(1 / 2)$ was the broadest subphase for any dipole moment $[1,6]$, therefore the new effect in the most cases could not "kill" it completely. Since the vertical polarization is proportional to the additional power of $\sin \theta$, its destructive effect is large only if the 


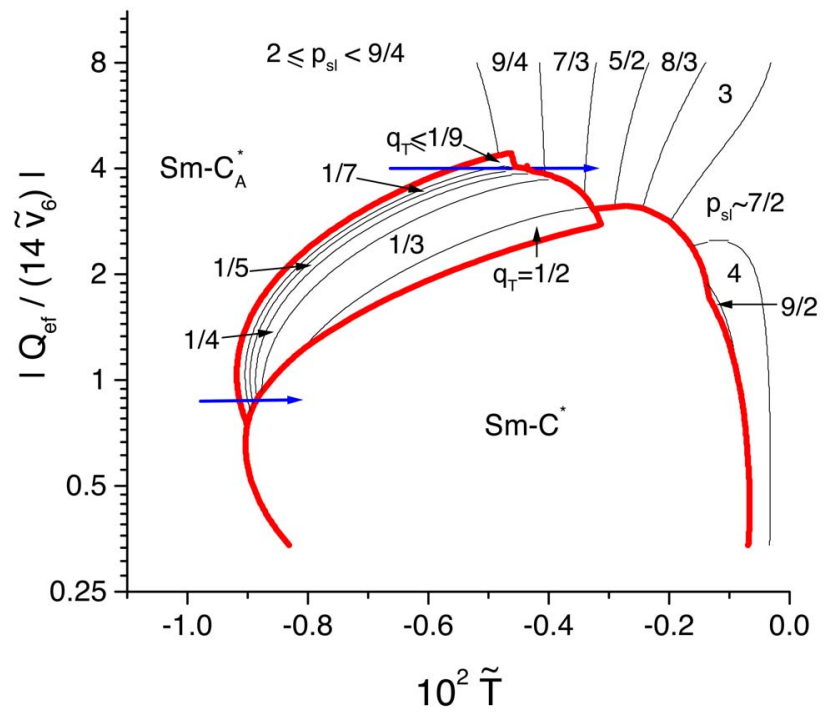

FIG. 7. (Color online) Reduced quadrupole-temperature phase diagram in the case $B_{0} / B_{1}=-0.1, \alpha / B_{1}=-0.5, \sigma=0.1, \ell / d=4, \widetilde{v}_{1}$ $=-0.622, \quad \Delta \widetilde{v}_{1}=0, \quad \widetilde{v}_{3}=1.71, \quad \widetilde{v}_{5}=-1.5 \times 10^{-2}, \Delta \widetilde{v}_{5}=5 \times 10^{-2}, \widetilde{v}_{6}$ $\times Q_{\mathrm{ef}}=-0.156 \times 10^{-2}$, and $\mu_{\mathrm{ef}}=0.77$.

tilt angle is large, and this corresponds to case 2 when almost no $\mathrm{Sm}-C_{A}^{*}(1 / 2)$ is observed.

The reduced quadrupole-temperature phase diagram (see Fig. 7) generally looks similar to the reduced dipoletemperature phase diagram. From both diagrams one notes another interesting effect: for the large values of the effective reduced dipole and quadrupole moments a situation is possible when subphase(s) with $q T<1 / 3$ are stable in a quite large temperature range, and neither $\mathrm{Sm}-C_{A}^{*}(1 / 2)$ nor $\mathrm{Sm}-C_{A}^{*}(1 / 3)$ is observed (see long blue arrow in Fig. 7). This reminds one of the recent experimental findings [7]. By definition, the biaxial subphases with $q_{T}<1 / 3$ look already very similar to each other, and according to our theory each one exists within the small temperature range, and thus, in the normal case [i.e., when $\mathrm{Sm}-C_{A}^{*}(1 / 2)$ and $\mathrm{Sm}-C_{A}^{*}(1 / 3)$ are observed] they may already be interpreted as pretransitional effects into $\mathrm{Sm}-C_{A}^{*}(0)$.

The helical pitch in various tilted smectic phases is presented in Fig. 8(a). One notes no big changes of this parameter between various subphases with $q_{T}<1 / 3$. At the same time, the difference between these subphases and Sm- $C_{A}^{*}(1 / 3)$ is rather noticeable. In [7] it was discussed that in pure 12BIMF10 only the new subphase with $q_{T}<1 / 3$ is observed. When mixing 12BIMF10 with a small percent of MHPBC first $\mathrm{Sm}-C_{A}^{*}(1 / 3)$ and then $\mathrm{Sm}-C_{A}^{*}(1 / 2)$ arise at higher temperatures. Generally, the phase sequence in 12BIMF10 seems to correspond the one represented by the long blue arrow in Fig. 7, and mixing with MHPBC seems to diminish the effective reduced quadrupole moment gradually. Another hypothetical possibility of existence of the biaxial subphases with $q_{T}<1 / 3$ and absence of $\mathrm{Sm}-C_{A}^{*}(1 / 3)$ and $C_{A}^{*}(1 / 2)$ is represented by the short blue arrow in Fig. 7 . This, however, does not seem to be the case reported in [7], because no $\mathrm{Sm}-C^{*}$ was observed there.

The helical pitch and the angle of 3D distortion from the Ising model in every tilted phase presented in Figs. 8(a) and
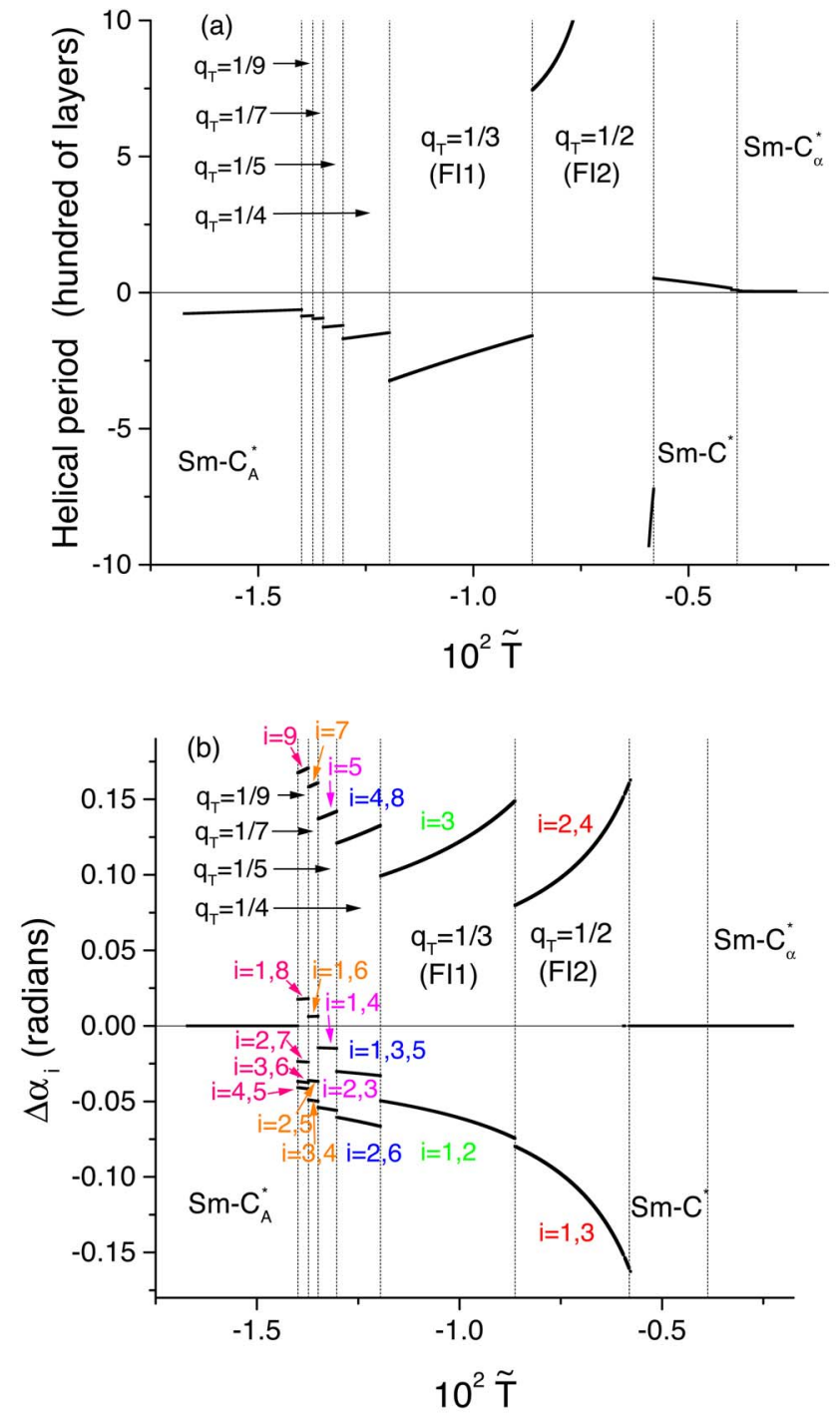

FIG. 8. (Color online) Helical period (a) and 3D distortion angle from Ising model (b) in different phases as functions of temperature in the case $B_{0} / B_{1}=-0.1, \alpha / B_{1}=-0.5, \sigma=0.2, \ell / d=4, \widetilde{v}_{1}=-0.622$, $\Delta \widetilde{v}_{1}=0, \widetilde{v}_{3}=1.67, \widetilde{v}_{5}=-1.5 \times 10^{-2}, \Delta \widetilde{v}_{5}=0, \widetilde{v}_{6}=-4.74 \times 10^{-2}, \mu_{\mathrm{ef}}$ $=0.77$, and $Q_{\mathrm{ef}}=3.29$.

8(b), respectively, as functions of temperature are estimated using the perturbation scheme (43). We have chosen negative conventional chirality $c_{1}=c_{2}$ and negative parameter $c_{p} c_{f}$ corresponding to the coupling between polar electrostatic and polar dispersion interactions and responsible for the combined chiral interaction. One notes that the helical rotation angle $\Delta \varphi$ and all the distortion angles $\Delta \alpha_{j}$ simply change their signs with the simultaneous change of signs of parameter $c_{p} c_{f}$ and of parameters $c_{1}$ and $c_{2}$. Usually the helical period in $\mathrm{Sm}-C^{*}$ and $\mathrm{Sm}-C_{A}^{*}$ has opposite signs, in every biaxial subphase with $q_{T} \leqslant 1 / 3$ the sign is the same as in $\mathrm{Sm}-C_{A}^{*}$, but the absolute value is larger. The helical rotation in $\mathrm{Sm}-C_{A}^{*}(1 / 2)$ is very weak (the helical period is very large and may diverge). Indeed, substituting $t=4$ into Eqs. (43)-(45) one obtains the following expressions for the distortion angle $\Delta \alpha_{4}^{1 / 2}$ and for the helical rotation angle $\Delta \varphi^{1 / 2}$ in $\mathrm{Sm}-C_{A}^{*}(1 / 2)$ : 


$$
\begin{aligned}
& \Delta \alpha_{4}^{1 / 2}=\frac{B_{2} C_{1}-B_{1} C_{2}}{B_{2} A_{1}-A_{2} B_{1}}, \\
& \Delta \varphi^{1 / 2}=\frac{A_{1} C_{2}-A_{2} C_{1}}{B_{2} A_{1}-A_{2} B_{1}},
\end{aligned}
$$

where

$$
\begin{gathered}
A_{1} \equiv 2 b \sin ^{2} \theta, \quad B_{1} \equiv\left(a-a_{1}\right) \cos ^{2} \theta+2 c_{f}^{2}, \\
C_{1} \equiv 4\left(\chi_{m}-1\right) c_{p} c_{f}+c_{1} \cos ^{2} \theta \\
A_{2} \equiv 2 a \cos ^{2} \theta, \quad C_{2} \equiv 2 c_{2} \sin ^{2} \theta, \\
B_{2} \equiv 4 b \sin ^{2} \theta-8 a_{1} \cos ^{2} \theta-16\left(\chi_{m}-1\right) c_{f}^{2} .
\end{gathered}
$$

One can check that parameter $a$ changes its sign from positive to negative somewhere in $\mathrm{Sm}-C_{A}^{*}(1 / 3)$ [very close to Sm- $\left.C_{A}^{*}(1 / 2)\right]$ with the increasing temperature, so that in $\mathrm{Sm}-C_{A}^{*}(1 / 2)$ it remains very small and negative. Thus in the absence of conventional chirality $\left(c_{1}=c_{2}=0\right)$ the rotation angle $\Delta \varphi^{1 / 2}$ appears to be very small and to have the same sign as $c_{p} c_{f}$ (negative in our case). Here we need to stress that additional terms proportional to $c_{p} c_{f}$ and independent of the small parameter $a$ disappeared from the expression for the rotation angle $\Delta \varphi^{1 / 2}$ due to the very special symmetry of Sm- $C_{A}^{*}(1 / 2)$, and only a similar term proportional to $c_{2}$ survived. It can be checked [by substituting different $t$ into Eqs. (43)-(45)] that in the other biaxial subphases an additional $c_{p} c_{f}$ term independent of $a$ is also present in the expression for $\Delta \varphi$, and therefore the helical rotation appears to be stronger. It is reasonable to suppose, however, that the conventional chirality is also nonzero and has the same sign as $c_{p} c_{f}$ (i.e., $\left.c_{1}=c_{2}<0\right)$. In this case a competition between two terms in the numerator of Eq. (53) for $\Delta \varphi^{1 / 2}$ arises, and the helical pitch diverges at some point within $\operatorname{Sm}-C_{A}^{*}(1 / 2)[$ see Fig. 8(a), where the helical period is very similar to the experimental one presented in [12] for 10OTBBB1M7]. Thus, experimentally, both conventional and combined chirality coefficients must be nonzero and of the same sign, that looks quite natural.

\section{CONCLUSIONS}

In this paper the general phase diagrams containing the entire set of the known fundamental and intermediate tilted smectic phases (including both biaxial and uniaxial ones) are presented in terms of the molecular parameters (in particular, the dipole and the quadrupole moments) and temperature. These diagrams demonstrate a surprising coincidence with the experimental results. They essentially contain the biaxial subphases with periodicity of more than four smectic layers which are extremely important for the further development of science and technology. Both increasing and decreasing with temperature periodicities of the uniaxial subphase are explained. An important role played by the vertical (perpendicular to the smectic layer plane) flexoelectric polarization is investigated for the first time. In particular, this polariza- tion destroys the four-layer biaxial subphase, so that in some cases this phase does not arise at all, and in most of the cases the three-layer biaxial subphase appears to dominate the other biaxial subphases. A perturbation scheme for the determination of the helical rotation in a strongly biaxial tilted smectic phase is suggested. The corresponding rotation angles are written analytically. A sharp transition from $\mathrm{Sm}-C^{*}$ to $\mathrm{Sm}-C_{\alpha}^{*}$ is explained in terms of the nonpolar biaxial ordering of molecules. The de Vries's phase is explained in terms of the entropy effects in the presence of the biaxial nonpolar ordering. The arguments are based on the molecular-statistical theory.

\section{ACKNOWLEDGMENTS}

The authors are grateful to M. A. Osipov whose idea about the discrete flexoelectric effect was essentially used in the present theory, and also to V. P. Panov, A. D. L. Chandani, and N. M. Shtykov for fruitful discussions. A.V.E. gratefully acknowledges the support of Russian Ministry of Science Grant MK-2034.2006.2 and of Russian Foundation for Basic Research Grant 04-03-32096. J.K.V. thanks the Science Foundation of Ireland for the Grant SFI (02/In.1/ I031) for funding the research and visit of A.V.E. to Dublin. A.F. acknowledges Trinity College Dublin for support [SFI (02/W/I02)].

\section{APPENDIX: CALCULATION OF THE AVERAGE DIPOLE- DIPOLE INTERACTION BETWEEN THE NEAREST MOLECULES LOCATED WITHIN THE SAME AND IN THE NEIGHBORING TILTED SMECTIC LAYERS}

The dipole-dipole interaction is determined by Eqs.(7) and (8), and in this section we are going to average it with respect to the positions of the interacting molecules. The most simple task is to average the dipole-dipole interaction between molecules located in the neighboring layers, because positionally correlated molecules in this case are situated just above each other, when the vector connecting dipole moments is parallel to the smectic layer normal $\mathbf{k}$, the minimal distance between them is equal to $h_{1} \cos \theta$ (see Fig. 1 ), and the average dipole-dipole interaction is simply equal to

$$
\left\langle U_{\mu \mu}^{i, i \pm 1}\right\rangle \approx\left\langle g_{i, i \pm 1}\right\rangle \mu_{\alpha}^{(i)} T_{\alpha \beta}\left(h_{1} \mathbf{k} \cos \theta\right) \mu_{\beta}^{(i \pm 1)},
$$

where $\left\langle g_{i, i \pm 1}\right\rangle$ is determined by Eq. (12), and

$$
T_{\alpha \beta}\left(h_{1} \mathbf{k} \cos \theta\right)=\frac{1}{h_{1}^{3} \cos ^{3} \theta}\left(\delta_{\alpha \beta}-3 k_{\alpha} k_{\beta}\right) .
$$

To average the dipole-dipole interaction between molecules located in the same smectic layer is a more difficult task, since the minimal distance between molecules (let us call it $r$ ) in this case depends on the orientation of the intermolecular vector $\mathbf{r}$ (which at the same time is the vector connecting the dipole moments):

$$
\left\langle U_{\mu \mu}^{i, i}\right\rangle \approx\left\langle g_{i, i \pm 1}\right\rangle \mu_{\alpha}^{(i)}\left\langle T_{\alpha \beta}(\mathbf{r})\right\rangle \mu_{\beta}^{(i)},
$$

where 


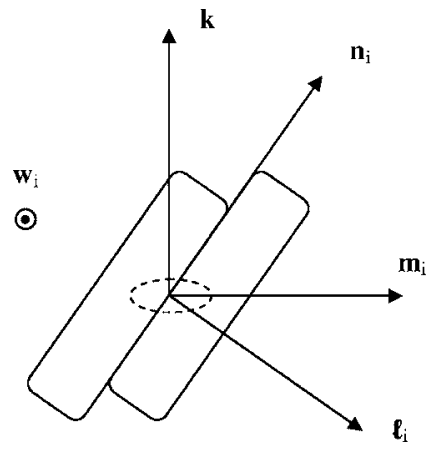

FIG. 9. Contacting molecules within the same smectic layer.

$$
\left\langle T_{\alpha \beta}(\mathbf{r})\right\rangle=\left\langle\frac{\delta_{\alpha \beta}}{r^{3}}-\frac{3 r_{\alpha} r_{\beta}}{r^{5}}\right\rangle .
$$

Vector $\mathbf{r}$ describes an ellipse with semiaxes $d$ and $d / \cos \theta$, and thus, its projections on axes $\mathbf{w}_{i}$ and $\mathbf{m}_{i}$ (see definitions in Sec. II and Fig. 9) obey the following equation:

$$
r_{w}^{2}+r_{m}^{2} \cos ^{2} \theta=d^{2}
$$

Let us introduce polar angle $\phi$ for vector $\mathbf{r}$ :

$$
r_{w}=r \cos \phi, \quad r_{m}=r \sin \phi .
$$

Substituting Eq. (A6) into Eq. ((A5)), one obtains

$$
r^{2}=d^{2} /\left(\cos ^{2} \phi+\cos ^{2} \theta \sin ^{2} \phi\right) .
$$

Using Eqs. (A6) and (A7), one can rewrite Eq. (A4) in the following form:

$$
\begin{aligned}
\left\langle T_{\alpha \beta}(\mathbf{r})\right\rangle= & \frac{1}{d^{3}} \frac{1}{s} \int_{0}^{2 \pi}\left(\cos ^{2} \phi+\cos ^{2} \theta \sin ^{2} \phi\right)^{3 / 2} \times\left[\delta_{\alpha \beta}\right. \\
& \left.-3 w_{\alpha}^{(i)} w_{\beta}^{(i)} \cos ^{2} \phi-3 m_{\alpha}^{(i)} m_{\beta}^{(i)} \sin ^{2} \phi\right] \frac{\partial s}{\partial \phi} d \phi,
\end{aligned}
$$

where we assumed that probability of contact for the two molecules is the same along the whole elliptic line, $s$ is the total length of this line, and $\partial s / \partial \phi$ is the Jackobian of transformation from variable position along the elliptic line to variable $\phi$. One notes that integral ((A8)) cannot be calculated exactly. Thus, let us estimate it by expanding all expressions in Taylor series and neglecting all the terms higher than quadratic in the small parameter $\theta$. Then the following approximation for the distance $r$ between molecules can be made [see Eq. $((\mathrm{A} 7))]$ :

$$
\frac{r}{d} \approx 1+\frac{1}{2} \theta^{2} \sin ^{2} \phi
$$

An infinitely small distance along the elliptic line can be expressed as

$$
d s=\sqrt{\left(d r_{w}\right)^{2}+\left(d r_{m}\right)^{2}} .
$$

Substituting Eq. (A9) into Eq. (A6), differentiating $r_{w}$ and $r_{m}$ with respect to $\phi$, and substituting the result into Eq. (A10), one obtains

$$
\frac{1}{d} \frac{\partial s}{\partial \phi} \approx 1+\frac{1}{2} \theta^{2} \sin ^{4} \phi-\frac{3}{2} \theta^{2} \sin ^{2} \phi \cos ^{2} \phi,
$$

and the total length of the elliptic line $s \approx 2 \pi d$ in the same approximation (it differs from $2 \pi d$ only in higher than quadratic terms in $\theta$ ). Substituting Eq. (A11) into Eq. (A8) and expanding the remaining terms in Eq. (A8) in Taylor series, one obtains

$$
\begin{aligned}
\left\langle T_{\alpha \beta}(\mathbf{r})\right\rangle \approx & \frac{1}{d^{3}}\left[\delta_{\alpha \beta}\left(1-\frac{3}{4} \theta^{2}\right)-\frac{3}{2} w_{\alpha}^{(i)} w_{\beta}^{(i)}\left(1-\frac{1}{2} \theta^{2}\right)\right. \\
& \left.-\frac{3}{2} m_{\alpha}^{(i)} m_{\beta}^{(i)}\left(1-\theta^{2}\right)\right] .
\end{aligned}
$$

Introducing a new vector $\ell_{i} \equiv\left[\mathbf{n}_{i} \times \mathbf{w}_{i}\right]$ (see Fig. 9), taking into account that $\mathbf{m}_{i}=\boldsymbol{\ell}_{i}+\mathbf{n}_{i} \tan \theta$, and using the following constraint for the three orthogonal unit vectors $\mathbf{n}_{i}, \mathbf{w}_{i}$, and $\ell_{i}$ :

$$
n_{\alpha}^{(i)} n_{\beta}^{(i)}+w_{\alpha}^{(i)} w_{\beta}^{(i)}+\ell_{\alpha}^{(i)} \ell_{\beta}^{(i)}=\delta_{\alpha \beta},
$$

one obtains

$$
\begin{aligned}
\left\langle T_{\alpha \beta}(\mathbf{r})\right\rangle \approx & -\frac{1}{2 d^{3}}\left[\delta_{\alpha \beta}\left(1-\frac{3}{2} \theta^{2}\right)+\frac{3}{2} w_{\alpha}^{(i)} w_{\beta}^{(i)} \theta^{2}-3 n_{\alpha}^{(i)} n_{\beta}^{(i)}(1\right. \\
& \left.\left.-2 \theta^{2}\right)\right]
\end{aligned}
$$

Substituting Eq. (A14) into Eq. (A3) one notes that the last term in Eq. (A14) plays no role, because $\left(\boldsymbol{\mu}_{i} \cdot \mathbf{n}_{i}\right)=0$. Finally, substituting Eqs. (A1)-(A3) and (A14) into Eq. (4) one obtains Eq. (9) with tensors $\hat{\mathbf{g}}_{0}$ and $\hat{\mathbf{g}}_{1}$ defined by Eq. (11).
[1] A. V. Emelyanenko and M. A. Osipov, Phys. Rev. E 68, 051703 (2003).

[2] A. V. Emelyanenko and M. A. Osipov, Ferroelectrics 309, 13 (2004)

[3] M. A. Osipov, A. Fukuda, and H. Hakoi, in Proceedings of Anglo-Japanese Joint Workshop held in Southampton, UK (Taylor and Francis, 2000) [Mol. Cryst. Liq. Cryst. 402, 9 (2003)].

[4] A. Fukuda, H. Hakoi, M. Sato, and M. A. Osipov, Mol. Cryst.
Liq. Cryst. 398, 169 (2003).

[5] M. Cepic and B. Zeks, Phys. Rev. Lett. 87, 085501 (2001).

[6] N. M. Shtykov, A. D. L. Chandani, A. V. Emelyanenko, A. Fukuda, and J. K. Vij, Phys. Rev. E 71, 021711 (2005).

[7] A. D. L. Chandani, N. M. Shtykov, V. P. Panov, A. V. Emelyanenko, A. Fukuda, and J. K. Vij, Phys. Rev. E 72, 041705 (2005).

[8] T. Isozaki, T. Fujikawa, H. Takezoe, A. Fukuda, T. Hagiwara, Y. Suzuki, and I. Kawamura, Jpn. J. Appl. Phys., Part 2 31, 
L1435 (1992).

[9] T. Isozaki, T. Fujikawa, H. Takezoe, A. Fukuda, T. Hagiwara, Y. Suzuki, and I. Kawamura, Phys. Rev. B 48, 13439 (1993).

[10] A. de Vries, Mol. Cryst. Liq. Cryst. 41L, 27 (1977).

[11] P. M. Johnson, D. A. Olson, S. Pankratz, T. Nguyen, J. Goodby, M. Hird, and C. C. Huang, Phys. Rev. Lett. 84, 4870 (2000).

[12] I. Musevic, M. Skarabot, G. Heppke, and H. T. Nguyen, Liq. Cryst. 29, 1565 (2002).

[13] M. A. Osipov and A. Fukuda, Phys. Rev. E 62, 3724 (2000).

[14] B. W. van der Meer and G. Vertogen, in Molecular Physics of Liquid Crystals, edited by G. R. Luckhurst and G. W. Gray (Academic Press, London, 1979).

[15] N. Vaupotic and M. Cepic, Phys. Rev. E 71, 041701 (2005).

[16] V. Laux, N. Isaert, G. Joly, and H. T. Nguyen, Liq. Cryst. 26, 361 (1999).
[17] C. D. Cruz, J. C. Rouillon, J. P. Marcerou, N. Isaert, and H. T. Nguyen, Liq. Cryst. 28, 125 (2001).

[18] L. S. Hirst, S. J. Watson, H. F. Gleeson, P. Cluzeau, P. Barois, R. Pindak, J. Pitney, A. Cady, P. M. Johnson, C. C. Huang, A.-M. Levelut, G. Srajer, J. Pollmann, W. Caliebe, A. Seed, M. R. Herbert, J. W. Goodby, and M. Hird, Phys. Rev. E 65, 041705 (2002).

[19] V. P. Panov, N. M. Shtykov, A. Fukuda, J. K. Vij, Y. Suzuki, R. A. Lewis, M. Hird, and J. W. Goodby, Phys. Rev. E 69, 060701(R) (2004).

[20] A. Cady, D. A. Olson, X. F. Han, H. T. Nguyen, and C. C. Huang, Phys. Rev. E 65, 030701(R) (2002).

[21] A. Cady, X. F. Han, D. A. Olson, H. Orihara, and C. C. Huang, Phys. Rev. Lett. 91, 125502 (2003).

[22] Yu. P. Panarin, O. Kalinovskaya, J. K. Vij, and J. W. Goodby, Phys. Rev. E 55, 4345 (1997). 